\title{
THE IRREGULAR SATELLITES: THE MOST COLLISIONALLY EVOLVED POPULATIONS IN THE SOLAR SYSTEM
}

\author{
William F. Bottke ${ }^{1}$, David Nesvorný ${ }^{1}$, David VokrouhlickÝ ${ }^{2}$, ANd Alessandro Morbidelli ${ }^{3}$ \\ ${ }^{1}$ Southwest Research Institute and NASA Lunar Science Institute 1050 Walnut Street Suite 300, Boulder, CO 80302, USA; bottke@ boulder.swri.edu \\ ${ }^{2}$ Institute of Astronomy, Charles University, V Holešovičkách 2, 18000 Prague 8, Czech Republic \\ ${ }^{3}$ Observatoire de la Côte d'Azur Boulevard de l'Observatoire, B.P. 4229, 06304 Nice, Cedex 4, France \\ Received 2009 May 28; accepted 2010 January 5; published 2010 February 2
}

\begin{abstract}
The known irregular satellites of the giant planets are dormant comet-like objects that reside on stable prograde and retrograde orbits in a realm where planetary perturbations are only slightly larger than solar ones. Their size distributions and total numbers are surprisingly comparable to one another, with the observed populations at Jupiter, Saturn, and Uranus having remarkably shallow power-law slopes for objects larger than $8-10 \mathrm{~km}$ in diameter. Recent modeling work indicates that they may have been dynamically captured during a violent reshuffling event of the giant planets $\sim 3.9$ billion years ago that led to the clearing of an enormous, $35 M_{\oplus}$ disk of comet-like objects (i.e., the Nice model). Multiple close encounters between the giant planets at this time allowed some scattered comets near the encounters to be captured via three-body reactions. This implies the irregular satellites should be closely related to other dormant comet-like populations that presumably were produced at the same time from the same disk of objects (e.g., Trojan asteroids, Kuiper Belt, scattered disk). A critical problem with this idea, however, is that the size distribution of the Trojan asteroids and other related populations do not look at all like the irregular satellites. Here we use numerical codes to investigate whether collisional evolution between the irregular satellites over the last $\sim 3.9$ Gyr is sufficient to explain this difference. Starting with Trojan asteroid-like size distributions and testing a range of physical properties, we found that our model irregular satellite populations literally self-destruct over hundreds of Myr and lose $\sim 99 \%$ of their starting mass. The survivors evolve to a low-mass size distribution similar to those observed, where they stay in steady state for billions of years. This explains why the different giant planet populations look like one another and provides more evidence that the Nice model may be viable. Our work also indicates that collisions produce $\sim 0.001$ lunar masses of dark dust at each giant planet, and that non-gravitational forces should drive most of it onto the outermost regular satellites. We argue that this scenario most easily explains the ubiquitous veneer of dark carbonaceous chondrite-like material seen on many prominent outer planet satellites (e.g., Callisto, Titan, Iapetus, Oberon, and Titania). Our model runs also provide strong indications that the irregular satellites were an important, perhaps even dominant, source of craters for many outer planet satellites.
\end{abstract}

Key words: comets: general - planets and satellites: formation - planets and satellites: general

\section{INTRODUCTION}

The irregular satellites are, in certain ways, the Oort Clouds of the giant planets. Like Oort Cloud comets, they are located far from their central object, making them denizens of a realm where external gravitational perturbations are only slightly smaller than the gravitational pull of the central body itself. The irregular satellites also have objects with eccentric and highly inclined (or even retrograde) orbits and spectroscopic signatures akin to dormant comets. Some even argue that the two populations had the same source location, namely, the primordial trans-planetary disk that was once located just beyond the orbits of the giant planets (Tsiganis et al. 2005).

The differences between the two populations, however, are just as profound. Oort Cloud comets reached their current orbits through a combination of scattering events by the giant planets and gravitational perturbations produced by passing stars and/ or galactic torques (see a review by Dones et al. 2004), while the irregular satellites were likely captured around the giant planets by a dynamical mechanism (see reviews by Jewitt \& Haghighipour 2007; and Nicholson et al. 2008). This means that the Oort comets currently reside in a distant storage zone where the odds of collisions between members are practically nil. The irregular satellites, on the other hand, were captured into a relatively tiny region of space with short orbital periods. This makes collisions between the objects almost unavoidable; collision probabilities between typical irregular satellites are generally four orders of magnitude higher than those found among main Belt asteroids, using the code described in Bottke et al. (1994). The analogy that comes to mind would be to compare the rate of car crashes occurring along the empty back roads of the American West to rush-hour traffic in Los Angeles.

Even though the irregular satellites have extremely high collision probabilities, the observed populations are small, such that collisional grinding among them at present is at a relatively low level. In fact, as we describe below, their size frequency distribution (SFD) for diameter $D>8 \mathrm{~km}$ objects is the most shallow yet found in the solar system (Figure 1; see Section 2.3). The populations are not in a classical Dohnanyilike collisional equilibrium with a differential power-law index of -3.5 (Dohnanyi 1969). Jewitt \& Sheppard (2005; see also Sheppard et al. 2006; Jewitt \& Haghighipour 2007; and Nicholson et al. 2008) also point out that once one accounts for observational selection effects, the combined prograde and retrograde irregular satellite populations at Jupiter-Neptune have surprisingly similar SFDs. They argue that this is unlikely to be a fluke, although the mechanism that produced this curious sameness is unknown. 


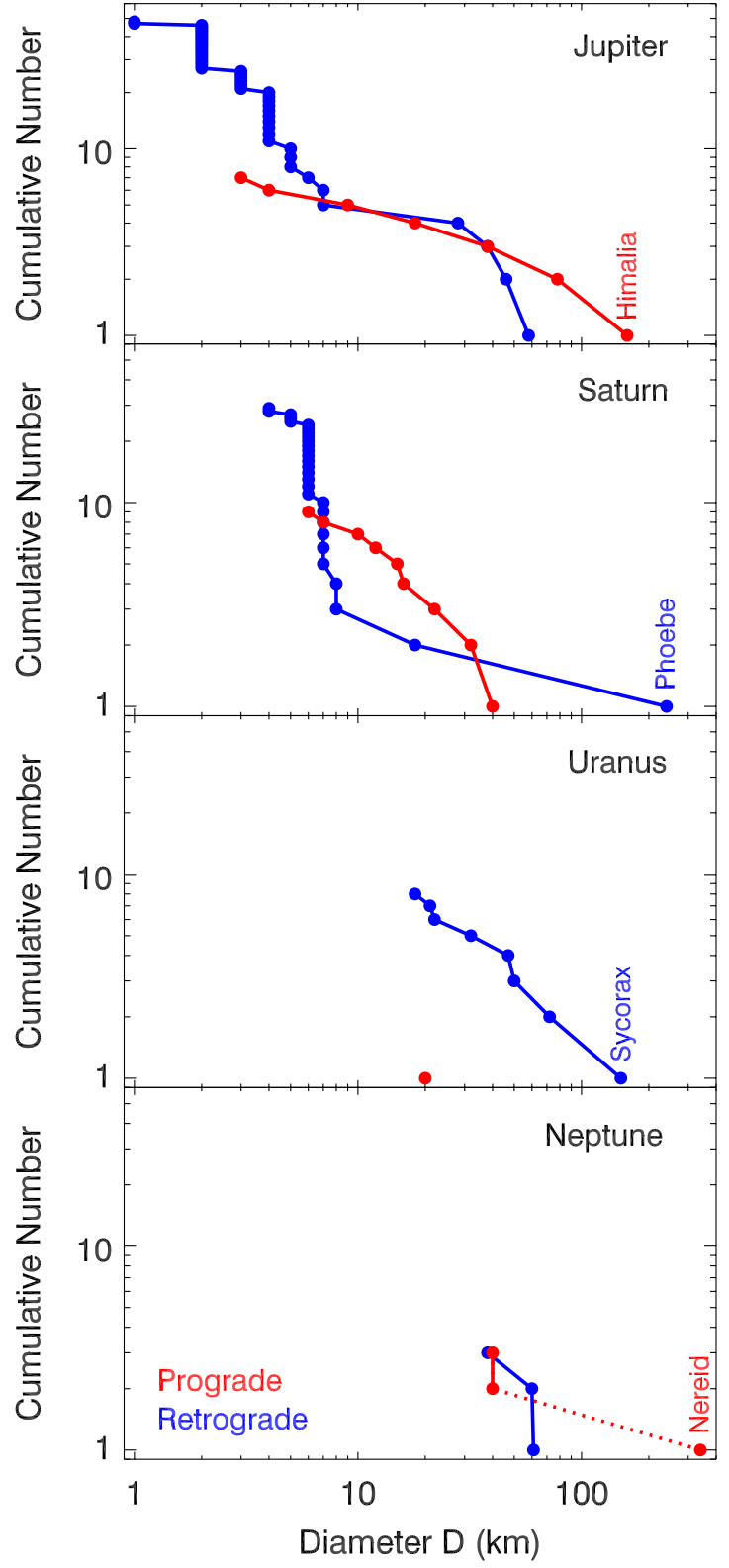

Figure 1. Cumulative SFD of the known prograde and retrograde irregular satellites at Jupiter, Saturn, Uranus, and Neptune as of 2009 May 1. The data on this plot are taken from Scott S. Sheppard's Jovian planet satellite Web site at http://www.dtm.ciw.edu/sheppard/satellites/. The prograde objects are in red and the retrograde objects are in blue.

It is possible that the shallow and similarly shaped SFDs are natural traits of the dynamical mechanism that left the irregular satellites in orbit around the giant planets. If true, the irregular satellite populations tell us something fundamental about the nature and evolution of objects in the primordial outer solar system. Coming up with a plausible capture mechanism that can meet these peculiar constraints, however, is extremely difficult. Recall that the giant planets formed in diverse regions of the solar nebula and are very different from one another (e.g., Jupiter and Saturn are 5-20 times more massive than Uranus and Neptune; Uranus and Neptune are compositionally more ice giants than gas giants).

Even recently proposed capture models run into trouble using SFDs as constraints. For example, Nesvorný et al. (2007) argued that irregular satellite capture may have taken place

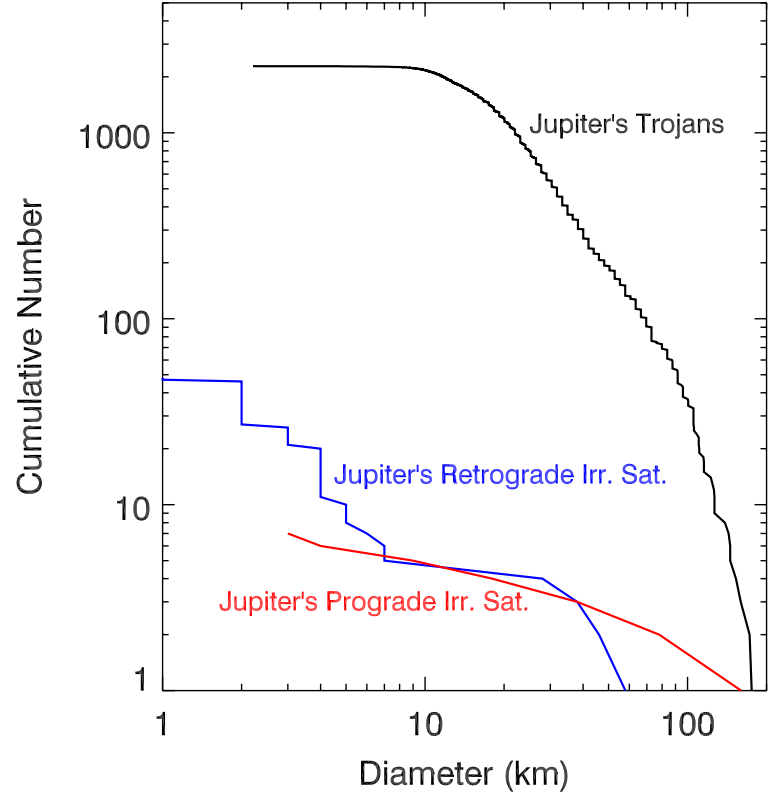

Figure 2. Cumulative SFD of Jupiter's known Trojan asteroids (the combined populations of $L_{4}$ and $L_{5}$ ) and the prograde and retrograde irregular satellites at Jupiter. The prograde objects are in red, and the retrograde objects are in blue. The absolute magnitude of the objects was converted to diameter using an albedo of 0.04 (e.g., Fernández et al. 2003). According to the framework of the Nice model described in Morbidelli et al. (2005), Nesvorný et al. (2007), and Levison et al. (2009), all of these populations were captured at approximately the same time from the same primordial trans-planetary disk. Based on this, we would expect the SFD of Jupiter's irregular satellites and Trojans immediately after capture to resemble one another. The fact that they are very different from one another indicates that the Nice model is wrong, at least as it is currently formulated, or that the irregular satellite population has had most of its mass removed by collisional evolution.

during giant planet close encounters within the so-called Nice model framework (Tsiganis et al. 2005; Morbidelli et al. 2005; Gomes et al. 2005; see below). Assuming they are right, the irregular satellite and Trojan SFDs, which were captured from the same source population at approximately the same time (Nesvorný et al. 2007; Morbidelli et al. 2009b; Levison et al. 2009), should look like one another (Figure 2; see Section 2.3). The fact that they are wildly different from one another might indicate the Nice model is wrong. Alternatively, given the high collision probabilities of the irregular satellites, it could be that the observed objects are the survivors of an extremely intense period of collisional evolution that took place near every giant planet. This scenario is no less interesting than the previous one because it would imply that the irregular satellite populations were initially much larger than we see today. Thus, by understanding their long-term evolution, we can glean insights into the physical properties of irregular satellites (and possibly comets themselves), their initial SFDs, and the nature of the conditions that once existed near different gas giants.

Taking this one step further, if we assume that the irregular satellite populations were once large, several intriguing implications come into play that may affect the regular giant planet satellites. For example, irregular satellites captured or moved onto unstable orbits may provide enough impactors to affect or perhaps even dominate the cratering records of the outer planet satellites. As a second example, consider that the collisional demolition of a large irregular satellite population would almost certainly produce a vast amount of primitive 
carbonaceous chondrite-like dust (e.g., perhaps similar to ground-up material from primitive meteorites like Orgueil or Tagish Lake). Some of this material would drift inward toward the central planet by non-gravitational forces, where it presumably could coat the surfaces of some regular satellites. We speculate that this dark debris would provide a natural way to explain the dark non-icy surface component found on the outermost satellites of Jupiter, Saturn, and Uranus (e.g., Ganymede/Callisto, Hyperion/Iapetus, Umbriel/Oberon). Accordingly, knowledge of the irregular satellites may help us understand the nature, evolution, and chronology of surface events on the regular satellites.

With this as motivation, we decided to use numerical simulations to explore whether the shallow SFDs and low masses of the irregular satellite populations could have been generated by collision evolution. Our initial conditions for this work are based on the numerical results of Nesvorný et al. (2007), who showed that objects from the primordial trans-planetary disk can be captured onto irregular satellite-like orbits during close encounters between the giant planets within the Nice model. For reference, the Nice model describes a period 3.9 Gyr when the giant planets experienced a violent reshuffling event that resulted in the depletion and/or scattering of the solar system's small body reservoirs (Tsiganis et al. 2005; Morbidelli et al. 2005; Gomes et al. 2005). The shape of the initial irregular satellite SFD in our model was chosen to resemble that of the present-day Trojan asteroids (Figure 2). Our rationale for this choice was that: (1) both populations originated in the primordial trans-planetary disk, (2) both were captured at approximately the same time within the Nice model framework, (3) the shape of the Trojan SFD strongly resembles that of trans-Neptunian objects (TNOs), both which probably came from the same primordial trans-planetary disk, and (4) the Trojans have experienced minimal collisional evolution over the last 3.9 Gyr for $D>10 \mathrm{~km}$ objects, a size range that covers many of the observed irregular satellites (Morbidelli et al. 2005, 2009b; Nesvorný et al. 2007; Levison et al. 2009).

Based on this, our work also serves as an ancillary test of the Nice model framework (i.e., if our assumed initial conditions cannot reproduce the observed irregular satellites, one could argue there is a flaw in the Nice model logic). More benignly, the constraints provided by our results should provide us with an additional means to constrain the events that took place $3.9 \mathrm{Gyr}$ ago in the outer solar system.

Note that the modeling work presented here cannot be used to rule out the possibility that one or more alternative populations or dynamical mechanisms were involved with the capture of at least some of the irregular satellites (e.g., perhaps the larger ones were asteroids captured by gas drag; Cuk \& Burns 2004). At the same time, however, we would argue that no alternative model is yet mature enough to permit adequate testing using our code; beyond a hypothesis, they would need to provide expected orbits and size distributions for their objects. The limitations of irregular satellite capture models in the literature are discussed in Section 3.1.

Our paper is organized as follows. In Section 2, we discuss what is known about the irregular satellites observed to date. Here we concentrate on irregular satellites orbiting Jupiter, Saturn, and Uranus, whose populations are complete or nearly so for diameter $D>10 \mathrm{~km}$. Note that we refrain from studying Neptune's irregular satellites at this time, partly because there are only a handful of known objects, all with $D>35 \mathrm{~km}$, but also because Triton's probable origin by binary capture
(Agnor \& Hamilton 2006) creates a number of interesting complications that warrant a closer look in a separate paper. In Section 3, we describe the irregular satellite capture mechanism of Nesvorný et al. (2007), our collisional evolution model Boulder, and the input parameters needed for our production runs. In Section 4, we show our results and demonstrate that the present-day irregular satellite populations found at Jupiter, Saturn, and Uranus were probably ground down from a much larger initial population. Finally, in Section 5, we summarize our results and discuss several implications of our work for the outermost regular satellites of Jupiter, Saturn, and Uranus.

\section{IRREGULAR SATELLITE CONSTRAINTS}

Here, we describe the orbital and physical characteristics of the irregular satellites relevant to our work. A more complete discussion of these topics can be found in recent review articles by Jewitt \& Haghighipour (2007) and Nicholson et al. (2008).

\subsection{Orbits}

The irregular satellites are defined as objects that orbit far enough away from their primary (i.e., Jupiter, Saturn, Uranus, and Neptune) that the precession of their orbital plane is controlled by solar rather than planetary perturbations. This distance was defined to be a semimajor axis $a>\left(2 \mu J_{2} R^{2} a_{p}^{3}\right)^{1 / 5}$, where $\mu$ is the ratio of the planet's mass to that of the Sun, $J_{2}$ is the planet's second zonal harmonic coefficient, $R$ is the planet's radius, and $a_{p}$ is the planet's semimajor axis (e.g., Burns 1986; Jewitt \& Haghighipour 2007).

The semimajor axes $a$ of the satellites around Jupiter to Neptune are more easily described if we scale them by their primary's Hill sphere, defined as $R_{\mathrm{H}}=a_{p}\left(m_{p} /\left(3 M_{\odot}\right)\right)^{1 / 3}$, where $m_{p}$ is the mass of the planet. Collectively, the irregular satellites have $a / R_{\mathrm{H}}$ values between $\sim 0.1$ and 0.5 . The prograde objects, however, have a smaller range of values $(0.1-0.3)$ than the retrograde ones $(0.2-0.5)$. Their eccentricity $e$ values range between 0.1 and 0.7 , with the retrograde satellites generally having larger $e$ values than the prograde ones. Their inclination $i$ values, to zeroth order, are similar to one another, with the prograde and retrograde populations avoiding $60^{\circ}<i<130^{\circ}$ where the Kozai resonance is active (Carruba et al. 2002, 2003; Nesvorný et al. 2003). Concentrations of irregular satellites in $(a, e, i)$ space, which are likely families produced by collision events (e.g., Nesvorný et al. 2004), will be discussed in greater detail below.

The orbital stability zones of the irregular satellites were determined numerically by Carruba et al. (2002) and Nesvorný et al. (2003; see also Beaugé et al. 2006). In general, these zones are modestly larger in $(a, e, i)$ space for retrograde objects than for prograde ones. The known irregular satellites fill a large fraction of these zones; there are no obvious voids that require explanation. This implies that the primordial satellite population was once larger, with objects captured onto nearly isotropic orbits. The objects injected onto unstable orbits, however, were either driven inward toward the primary, where they collided with the primary or the primary's regular satellites, or outward to escape beyond the planet's Hill sphere. Numerical simulations indicate that the majority of these entered the zone of the regular satellites (and were presumably lost by collisions) within $<10^{4}$ yr of capture. 


\subsection{Colors}

The irregular satellites have colors that are consistent with dark C-, P-, and D-type asteroids that are prominent in the outer asteroid Belt and dominate the Hilda and Trojan asteroid populations (e.g., Grav et al. 2003, 2004; Grav \& Bauer 2007; Grav \& Holman 2004). Spectrally, these objects are a good match to the observed dormant comets. Levison et al. (2009) claim that this may not be a coincidence and use numerical simulations to suggest and that the aforementioned populations may be captured refugees from the massive primordial transplanetary disk that once existed beyond the orbit of Neptune (see also Morbidelli et al. 2005). This issue will be discussed in greater detail below.

\subsection{Size-Frequency Distributions}

The cumulative SFDs of the irregular satellites are shown in Figure 1. The data on this plot, taken from Scott S. Sheppard's Jovian planet satellite Web site at http:// www.dtm.ciw.edu/sheppard/satellites/, contain what is known of the irregular satellite population as of 2009 May 1. Jupiter has 55 irregular satellites ( 7 prograde, 48 retrograde), Saturn has 38 (9 prograde, 29 retrograde), Uranus has 9 (1 prograde, 8 retrograde), and Neptune has 6 (3 prograde, if one counts Nereid, and 3 retrograde). The decrease in satellite numbers as one moves further from the Sun corresponds to the increasing difficulty observers have in detecting small dark distant objects (e.g., Sheppard et al. 2006). The satellite diameters were computed using Equation (2) of Sheppard et al. (2005, 2006) under the assumption that the objects have albedos of 0.04 , values that are typical for dormant comets (e.g., Jewitt 1991) and C-, D-, and P-type asteroids (e.g., Fernández et al. 2003).

It is important to point out here that in terms of detection and discovery, irregular satellites are different from asteroids or TNOs. For the latter, sky coverage, distance from opposition and the observer, recovery, etc. are all important issues that vary from survey to survey. For irregular satellites, however, the Hill spheres for planets like Saturn, Uranus, and Neptune have been completely covered by observations down to the limiting magnitude of the specific survey (e.g., Gladman et al. 2001; Sheppard et al. 2005, 2006). Accordingly, observational incompleteness in each population does not gradually fall off but instead is nearly a step function of magnitude. In other words, the satellites are close to $100 \%$ complete down to magnitude $M_{1}$ and close to $100 \%$ incomplete for magnitude $M>M_{2}=M_{1}+d M$, where $d M$ is small. Values of $M_{1}$ and $M_{2}$ are discussed for individual planets in various irregular satellite detection papers (a list of useful references can be found in Jewitt \& Haghighipour 2007 and Nicholson et al. 2008).

Note that Jupiter's Hill sphere has yet to be fully covered, so completeness is not yet at $100 \%$. As described in the introduction, it has also been argued that the prograde and retrograde SFDs, when combined together, are surprisingly similar to one another, particularly when observational incompleteness at Uranus and Neptune is considered (Jewitt \& Sheppard 2005; see reviews in Jewitt \& Haghighipour 2007 and Nicholson et al. 2008). The combined prograde and retrograde SFDs at each planet have a cumulative power-law index of $q \approx-1$ for $20<D<200 \mathrm{~km}$ (i.e., cumulative number $\left.N(>D) \propto D^{q}\right)$. Using the data from Figure 1, we find that this value also holds, more or less, down to $D>8 \mathrm{~km}$ objects $(q \approx-0.9)$, though Jupiter and Saturn have shallower slopes over this size range ( $q \approx-0.8$ and -0.7 , respectively), and the prograde population for Saturn has a steeper power-law slope for $D>8 \mathrm{~km}$ objects than the retrograde ones. The retrograde SFDs for $D<8 \mathrm{~km}$ bodies at Jupiter and Saturn, where we have data, then steepen up. The steepest slope, $q=-3.3$, is found for Saturn's retrograde satellites between $6<D<8 \mathrm{~km}$.

Other small body populations, measured over the same size ranges as those described above, cannot rival the shallow powerlaw slopes seen among the irregular satellites. Instead, most have $q$ values near -2 ; the outer main Belt, Hilda, and Trojan SFDs for $20<D<100 \mathrm{~km}$ all have $q \approx-2$ (e.g., Figure 2; see also Levison et al. 2009), the main Belt and near-Earth object (NEO) populations for $1<D<10 \mathrm{~km}$ is from -1.8 to -2.0 (e.g., Bottke et al. 2002, 2005a, 2005b; Stuart $\&$ Binzel 2004), and the ecliptic comet SFD for $D>3 \mathrm{~km}$ is $q=-1.9 \pm 0.3$ (see the review by Lamy et al. 2004). Even fading or dormant comet populations, which are or have been strongly affected by mass-loss mechanisms and disruption events (e.g., Levison et al. 2002), do not quite reach the shallow slopes of the irregular satellites. Faint Jupiter-family comets (JFCs) that become NEOs and achieve perihelion $<1.3 \mathrm{AU}$ have both a steep branch for bright comets $(q=-3.3 \pm 0.7$ for absolute comet nuclear magnitudes $\left.H_{10}<9\right)$ and a shallow branch for dimmer comets $\left(q=-1.3 \pm 0.3\right.$ for $9<H_{10}<18$; Fernández \& Morbidelli 2006). Most observed dormant comets have sizes between $2 \mathrm{~km}<D<20 \mathrm{~km}$. Those classified as JFCs have $q=-1.5 \pm 0.3$ (Whitman et al. 2006), while those classified as external returning comets and Halley-type comets have $q=-1.4$ or perhaps even $q=-1.2$, depending on the modeling parameters used (Levison et al. 2002). Thus, we conclude the irregular satellite for $D>8 \mathrm{~km}$ has some of the shallowest, if not the shallowest, SFDs in the solar system.

While we find the similarities intriguing, the various SFDs also have distinct differences that may provide important clues to their origins. For example: (1) Jupiter's retrograde SFD for $D<8 \mathrm{~km}$ is shallower than Saturn's, while the shapes of their prograde SFDs are very different from one another. (2) Uranus's prograde population is limited to a single $D>20 \mathrm{~km}$ object, while those for Jupiter and Saturn have 3-4 such objects. (3) There is nearly an order of magnitude difference in diameter between Phoebe and the second largest retrograde irregular satellite of Saturn. No comparable size difference can be found among any sub-population in Figure 1 unless one counts the size difference between Nereid, which may be a regular satellite that was scattered during the capture of Triton (Goldreich et al. 1989; Banfield \& Murray 1992; Agnor \& Hamilton 2006), and its two prograde brethren. (4) Jupiter's and Saturn's prograde populations are comparable to or significantly larger than their retrograde ones for $D>8 \mathrm{~km}$.

\subsection{Families}

Irregular satellite families are clusters of objects with similar proper $(a, e, i)$ parameters with respect to the primary planet. They are produced by cratering or catastrophic impact events, the latter defined as an impact event where $50 \%$ of the mass is ejected at escape velocity from the parent body.

Families appear to be an important component of the inventory of irregular satellite populations and can be identified once several tens of objects have been found. Among Jupiter's irregular satellites, Nesvorný et al. (2003, 2004) identified two robust retrograde families using clustering techniques and numerical integration simulations. They are the Carme family, which includes $D=46 \mathrm{~km}$ Carme and 13 members with $D=1-5 \mathrm{~km}$, and the Ananke family, which includes $D=28 \mathrm{~km}$ Ananke 
and 7 members with $D=3-7 \mathrm{~km}$. Himalia, a prograde $D=$ $160 \mathrm{~km}$ satellite, may also be in a family of four objects with $D=4-78 \mathrm{~km}$. When combined, these families represent about half of Jupiter's known prograde and retrograde satellites. For additional details, see Beaugé \& Nesvorný (2007).

A potential problem with the Himalia family is that the dispersion velocity between the members is significantly larger than those inferred for well-studied main Belt asteroid families (Bottke et al. 2001; Nesvorný et al. 2003). One possible solution for this was identified by Christou (2005), who used numerical integration simulations to show that the large dispersion velocities of three members (with $D=4-38 \mathrm{~km}$ ) could have been produced by numerous close encounters with Himalia itself. If true, some irregular satellites families may be too dispersed to find by clustering algorithms alone. A second potential solution with implications for all irregular satellites is discussed below.

At Saturn, Gladman et al. (2001) identified two prograde groups orbiting Saturn that contain three and four objects, respectively, with $D=10-40 \mathrm{~km}$. If real, these groups constitute $\sim 80 \%$ (seven of nine) of Saturn's known prograde satellites. A retrograde group of four objects with $D=7-18 \mathrm{~km}$ has been linked to Phoebe $(D=240 \mathrm{~km})$, though like the Himalia family the dispersion velocities between Phoebe and its putative members are larger than those found among main Belt families (Nesvorný et al. 2003, 2004). If high dispersion velocities are common among irregular satellite families with large parent bodies, perhaps produced by mutual close encounters (e.g., Christou 2005) or some other mechanism (see below), the Phoebe family could have many more members. Thus, like Jupiter, collisional families are likely an important, perhaps dominant, component of Saturn's irregular satellite population.

The irregular satellite populations around both Uranus and Neptune have less than 10 members, too few to probe for families in a meaningful way.

Ideally, we would like to use irregular satellite families to constrain the collisional evolution of irregular satellite systems. After some consideration, however, we decided to shy away from doing so in this paper. Our rationale is that the study of family-forming events among irregular satellites is far less mature than those among main Belt asteroids. At present, it is fair to say that no one yet understands the ejecta mass-velocity distribution function produced by irregular satellites or comet impact events.

To illustrate this complicated and somewhat bizarre issue, consider that while $\mathrm{P} / \mathrm{D}$-types are fairly common beyond the outer main Belt, we have yet to identify a robust P/D-type family anywhere in the solar system. Tests indicate P/D-types do not exist in the outer main Belt (Mothé-Diniz \& Nesvorny 2008) nor the Hilda/Trojan populations (Brož \& Vokrouhlický 2008). For the latter, Brož \& Vokrouhlický (2008) investigated the 1200 known Hildas and 2400 known Trojans for asteroid families. Despite the fact that $90 \%$ of the Hildas/Trojans are D/P-types, only C-type families were found; two robust families produced by $D>100 \mathrm{~km}$ disruption events in the Hilda population and one in the Trojan population. For reference, C-type asteroids have flat, somewhat nondescript spectra and are common across the main asteroid Belt.

At present, we have only begun to explore the physics of impacts into primitive volatile-rich highly porous materials. Preliminary experimental results indicate that when high-velocity projectiles are shot into highly porous targets, impact-generated shock waves may heat up porous material so much that, although the target is under pressure and is in compression, the density decreases because of extensive heat production and resulting thermal expansion (e.g., Holsapple \& Housen 2009). This effect could eject fragments at much higher speeds than those inferred from asteroid family-forming events. In turn, this would prevent standard clustering algorithms from finding all of the important members of a family, particularly when few objects are known. All in all, this could provide the easiest solution to the Himalia family conundrum described above. It is even possible that some impact events among irregular satellites launch fragments onto unstable orbits where they could strike the regular satellites (see Section 5.3).

For these reasons, in this paper we refrain from using irregular satellite families as serious constraints for our collisional evolution model.

\subsection{Summary}

We infer that while the irregular satellites share many of the same physical characteristics as other small solar system bodies, they evolved in dramatically different ways. Our key points from the data are as follows.

1. The irregular satellites were captured on nearly isotropic orbits and were once more numerous than they are today. The size of the initial population is unknown.

2. The $D>8 \mathrm{~km}$ objects have shallow slopes unlike any small body population yet observed in the solar system. This implies that they were affected by mechanisms in a manner and/or to a degree unlike the C-, P-, and D-type bodies found among the outer main Belt, Hilda, and Trojan asteroid populations or the active/dormant comets.

3. The prograde and retrograde satellite SFDs for each planet are similar in certain ways (e.g., population size, overall shape of SFDs) but are distinctly different in other ways (e.g., size and shape of the prograde versus retrograde populations). This makes it difficult to characterize the SFDs without understanding how they reached this point in their evolution.

4. Families are an important component of observed irregular satellite populations with several tens of members (i.e., Jupiter, Saturn). We infer from this that impacts have been a key factor in the evolution of the irregular satellite populations. Our understanding of the impact physics controlling irregular family-forming events, however, is still in its infancy.

\section{MODELING THE EVOLUTION OF THE IRREGULAR SATELLITES}

\subsection{Introduction and Motivation}

Nearly all recent papers discussing the origin of the irregular satellites have two sections in common: a section detailing previous attempts to explain the capture of planetesimals from heliocentric to observed irregular satellite orbits and a second section describing the deficiencies of those models. Accordingly, we will try to keep our discussion of these issues brief. The main capture scenarios described to date are: (1) capture by collisions between planetesimals (Colombo \& Franklin 1971; Estrada \& Mosqueira 2006); (2) capture due to the sudden growth of the gas giant planets, which is often referred to as the "pull-down" capture method (Heppenheimer \& Porco 1977), (3) capture of planetesimals due to the dissipation of their orbital energy via gas drag (Pollack et al. 1979; Astakhov et al. 2003; Ćuk \& Burns 2004; Kortenkamp 2005), (4) capture 
during resonance-crossing events between primary planets at a time when gas drag was still active (Ćuk \& Gladman 2006); (5) capture in three-body exchange reactions between a binary planetesimal and the primary planet (Agnor \& Hamilton 2006; Vokrouhlický et al. 2008), and (6) capture in three-body interactions during close encounters between the gas giant planets within the framework of the so-called Nice model (Nesvorný et al. 2007, and see below).

The problems with most of these models are discussed in several papers; recent summaries can be found in Jewitt \& Haghighipour (2007), Nesvorný et al. (2007), Nicholson et al. (2008), and Vokrouhlický et al. (2008). Essentially, all of these models (except possibly step (6); see below) are unsatisfying at some level because they suffer from one or more of the following problems: they are underdeveloped, they are inconsistent with what we know about planet formation processes and/or planetary physics, their capture efficiency is too low to be viable, they require exquisite and probably unrealistic timing in terms of gas accretion processes or the turning on/off of gas drag, they cannot reproduce the observed orbits of the irregular satellites, and they can produce satellites around some but not all gas giants. Moreover, there is the newly recognized problem that if the outer planets migrate after the capture of the irregular satellites, the satellites themselves will be efficiently removed by the passage of larger planetesimals or planets through the satellite system. This means that while different generations of irregular satellites may have existed at different times, the irregular satellites observed today were probably captured relatively late in a gas-free environment.

We argue here that the model with the fewest problems is No. 6 from Nesvorný et al. (2007), with late planetary migration acting not as a "sink" but rather as the conduit for satellite capture. In their scenario, migration leads to close encounters between pairs of gas giant planets over an interval of several millions of years. This allows planetesimals wandering in the vicinity of the encounter site to become trapped onto permanent orbits around the gas giants via gravitational threebody reactions.

To get gas giant close encounters, Nesvorný et al. (2007) invoked the so-called Nice model framework (Tsiganis et al. 2005; Morbidelli et al. 2005; Gomes et al. 2005) that assumes the Jovian planets experienced a violent reshuffling event in the past (presumably $\sim 3.9 \mathrm{Ga}$ ). The starting assumption for the Nice model is that the Jovian planets formed in a more compact configuration than they have today, with all located between $5 \mathrm{AU}$ and $15 \mathrm{AU}$. Slow planetary migration was induced in the Jovian planets by gravitational interactions with planetesimals leaking out of a $\sim 35 M_{\oplus}$ planetesimal disk residing between $\sim 16 \mathrm{AU}$ and $30 \mathrm{AU}$ (i.e., known as the primordial trans-planetary disk). Eventually, after a delay of $\sim 600 \mathrm{Myr}(\sim 3.9 \mathrm{Ga})$, Jupiter and Saturn crossed a mutual mean motion resonance. This event triggered a global instability that led to a reorganization of the outer solar system; planets moved and in some cases had close encounters with one another, existing small body reservoirs were depleted or eliminated, and new reservoirs were created in distinct locations.

Despite its radical nature, the Nice model has been successfully used to deal with several long-standing solar system dynamics problems. It can quantitatively explain the orbits of the Jovian planets (Tsiganis et al. 2005), a partial clearing of the main Belt via sweeping resonances (Levison et al. 2001; Minton \& Malhotra 2009), and the likely occurrence of a terminal cataclysm on the Moon and other terrestrial planets (Gomes et al.
2005; Strom et al. 2005). The timing of the Nice model would then be linked to the formation time of late forming lunar basins like Serentatis and Imbrium $\sim 3.9$ Ga (e.g., Stöffler \& Ryder 2001; Bottke et al. 2007). As we will show below, however, the precise timing of the Nice model does not play a large role in determining our results.

Perhaps the most critical test of the Nice model is determining what happens to objects from the primordial trans-planetary disk. According to simulations, giant planet migration led to resonance migration and resonance-crossing events that allowed a small fraction of scattered disk objects to be captured within the outer main Belt, Hilda, Jupiter and Neptune Trojan regions, irregular satellites, and TNO regions (Morbidelli et al. 2005, 2009b; Tsiganis et al. 2005; Nesvorný et al. 2007; Levison et al. 2009; Nesvorný \& Vokrouhlický 2009). Thus, the comet-like bodies in these populations all came from the same reservoir and thus should presumably have similar properties.

This prediction appears to hold true from a taxonomy standpoint. The observed small objects in these populations are mainly dormant comet-like objects (i.e., C-, D-, and P-type bodies). We cannot yet rule out the possibility, however, that majority of small planetesimals beyond 2.8 AU formed this way.

More compellingly, this scenario also seems to work from a SFD perspective. Morbidelli et al. (2009b) showed the SFD of the TNOs is similar to that of Jupiter's Trojans, while Levison et al. (2009) showed that the D/P-type objects in the outer main Belt, Hilda, Trojan populations could all have come from a source population with a SFD shaped like the present-day Trojan asteroids. Sheppard \& Trujillo (2008) also find that the cumulative luminosity function of the Neptune Trojans may have a turnover at the same place as the Trojans and Kuiper Belt objects (i.e., if converted into diameter, the turnover occurs near $D \sim 100 \mathrm{~km}$ ). Thus, it is unavoidable; if Nesvorný et al. (2007) are correct and the irregular satellites came from the primordial trans-planetary disk, they should have the same starting population as the Trojans (Figure 2).

Given all this, we will base our calculations on the Nice model framework and will test whether the irregular satellites could have come from an SFD that originally had the same shape as the Trojan asteroids.

As a caveat, we point out that some irregular satellite capture scenarios cannot yet be ruled out based on the modeling done below. It is possible that some of them, if proven true, could change our initial conditions (e.g., sweeping resonances may have slightly modified the nature of the orbital populations; Ćuk \& Gladman 2006). At this time, however, we believe no other scenario is mature enough to test within our model.

\subsection{Details of Irregular Satellite Capture Within the Nice Model}

Nesvorný et al. (2007) modeled satellite capture in several steps. In step 1, they created synthetic Nice model simulations that examined the time shortly after Jupiter and Saturn had migrated through the 2:1 mean motion resonance and initiated the violent reshuffling event. The initial conditions of the gas giants were taken from the Gomes et al. (2005) simulations, with Jupiter, Saturn, Uranus, and Neptune at 5.4 AU, 8.4 AU, 12.3 AU, and $18 \mathrm{AU}$. The primordial trans-planetary disk was filled with thousands of planetesimals between $21 \mathrm{AU}$ and $35 \mathrm{AU}$ and were given a variety of initial configurations. These systems were tracked for at least $130 \mathrm{Myr}$ using the symplectic integrator 
SyMBA (Duncan et al. 1998), with close encounters between the gas giants recorded for later use.

In step 2, they sifted the results for planetary systems that resembled our own and then used recorded gas giant close encounter and planetesimal/planet orbital data to create a high-fidelity satellite capture model. First, the orbits of the planetesimals and planets from step 1 at the time of each gas giant close encounter were integrated slightly backward in time to a time just before the encounter. Next, they used the planetesimals to construct orbital distribution maps suitable for creating millions of new test bodies on planetesimal-like orbits. Then, the test bodies and planets were integrated forward in time all the way through the close encounter. Objects deflected by the close encounter into planet-bound orbits were integrated for stability. Those satellites that survived were then followed into the next gas giant encounter, where their orbits could change or they could even be stripped altogether from their primary. This process was repeated until all close encounters within each run were completed.

Overall, Nesvorný et al. (2007) found that planetary encounters can create irregular satellites around Saturn, Uranus, and Neptune with $(a, e, i)$ distributions that are largely similar to the observed ones. A drawback, however, is that Jupiter does not generally participate in close encounters in the Nice model (Tsiganis et al. 2005; Gomes et al. 2005), such that only certain runs were capable of producing Jupiter's irregular satellites. Rather than representing a fundamental flaw in the work, we instead consider this to be an indication that we are still missing some important aspects in our understanding of the Nice model itself. For example, Brasser et al. (2009) find that close encounters between Jupiter and the ice giants are needed to prevent the terrestrial planets from obtaining high eccentricities via secular resonance sweeping. It is also possible that the gas giants were started in a different and even more compact configuration, with the instability triggered by a different resonance (see Morbidelli \& Crida 2007; Morbidelli et al. 2007). Regardless, the success of the Nesvorný et al. model for Saturn, Uranus, and Neptune and the orbital similarity of Jupiter's irregular satellite system to the other systems implies to us that Jupiter's irregular satellites were produced by gas giant encounters. On this basis, we will assume that the irregular satellites of all of the planets can be modeled using the Nesvorný et al. (2007) template.

\subsection{Collisional Evolution Code}

Our collisional modeling simulations will employ Boulder, a new code capable of simulating the collisional fragmentation of multiple planetesimal populations using a statistical particlein-the-box approach. A full description of the code, how it was tested, and its application to both accretion and collisional evolution of the early asteroid Belt, can be found in Morbidelli et al. (2009a). Examples of its use for the outer main Belt, Hildas, Trojans, and primordial trans-planetary disk can be found in Levison et al. (2009). Boulder was constructed along the lines of comparable codes (e.g., Weidenschilling et al. 1997; Kenyon \& Bromley 2001) and can be considered an updated and more flexible version of the well-tested collisional evolution and dynamical depletion model code CoDDEM used by Bottke et al. (2005a, 2005b) to simulate the history of the main Belt.

A major difference between Boulder and other codes like CoDDEM is that Boulder uses the results of smoothed particle hydrodynamics (SPH) $/ N$-body impact experiments to model the fragment SFD produced in asteroid disruption events (Durda et al. 2004, 2007). This is a key factor because, as we will show, accurate descriptions of different kinds of impact events are needed to model the irregular satellite SFDs. The code's procedure for modeling an impact is as follows.

For a given impact between a projectile and a target object, the code computes the impact energy $Q$, the kinetic energy of the projectile per unit mass of the target, and the critical impact specific energy $Q_{D}^{*}$, defined as the energy per unit target mass needed to disrupt the target and send $50 \%$ of its mass away at escape velocity (e.g., Davis et al. 2002). For reference, $Q<Q_{D}^{*}$ events correspond to cratering events, $Q \approx Q_{D}^{*}$ events correspond to barely catastrophic disruption events, and $Q>Q_{D}^{*}$ events correspond to super-catastrophic disruption events.

Numerical hydrocode experiments show that the mass of the largest remnant after a collision follows a linear function of $Q / Q_{D}^{*}$ (Benz \& Asphaug 1999), with the mass of the largest remnant $\left(M_{\mathrm{LR}}\right)$ produced by a given impact:

$$
\begin{aligned}
& M_{\mathrm{LR}}\left(Q<Q_{D}^{*}\right)=\left[-\frac{1}{2}\left(\frac{Q}{Q_{D}^{*}}-1\right)+\frac{1}{2}\right] M_{T}, \\
& M_{\mathrm{LR}}\left(Q>Q_{D}^{*}\right)=\left[-0.35\left(\frac{Q}{Q_{D}^{*}}-1\right)+\frac{1}{2}\right] M_{T},
\end{aligned}
$$

where $M_{T}$ is the target mass.

To choose the fragment SFD ejected from the collision, we take advantage of data derived from the numerical hydrocode impact experiments of Durda et al. (2004, 2007). As part of a project to study asteroid satellite formation, Durda et al. (2004) used smooth particle hydrodynamic (SPH) codes coupled with $\mathrm{N}$-body codes to perform 160 numerical impact experiments where they tracked $D=10-46 \mathrm{~km}$ projectiles slamming into $D=100 \mathrm{~km}$ basaltic spheres at a wide range of impact speeds $\left(2.5-7 \mathrm{~km} \mathrm{~s}^{-1}\right)$ and impact angles $\left(15^{\circ}-75^{\circ}\right)$. Durda et al. (2007) reported that the SFDs produced by these simulations, when scaled to the appropriate parent body size, were a good match to the SFD of the largest members of many observed asteroid families (i.e., the largest bodies had not been seriously affected by comminution since their formation; Bottke et al. 2005a, 2005b).

To include these results in Boulder, the mass of the largest fragment and the slope of the power-law SFD for each of the Durda et al. experiments were tabulated as a function of the ratio $Q / Q_{D}^{*}$. Empirical fits to the experimental data indicated that the mass of the largest fragment $\left(M_{\mathrm{LF}}\right)$ and slope of the cumulative power-law size distribution of the fragments $(q)$ could be written as

$$
\begin{aligned}
M_{\mathrm{LF}} & =8 \times 10^{-3}\left[\frac{Q}{Q_{D}^{*}} \exp ^{-\left(\frac{Q}{4 Q_{D}^{*}}\right)^{2}}\right] M_{\mathrm{tot}}, \\
q & =-10+7\left(\frac{Q}{Q_{D}^{*}}\right)^{0.4} e^{-\frac{Q}{7 Q_{D}^{*}}}
\end{aligned}
$$

with $M_{\text {tot }}$ being the combined mass of the projectile and target bodies.

Using these equations, Boulder selects a largest remnant, a largest fragment, and the exponent of the power-law fragment SFD. This allows it to accurately treat both cratering and supercatastrophic disruption events in a realistic manner.

Note that in some extreme cases, such as like-sized bodies smashing into one another, $M_{\mathrm{LF}}>M_{\mathrm{LR}}$. This describes a highly energetic super-catastrophic event capable of pulverizing both the projectile and target bodies. For the runs described here, we 


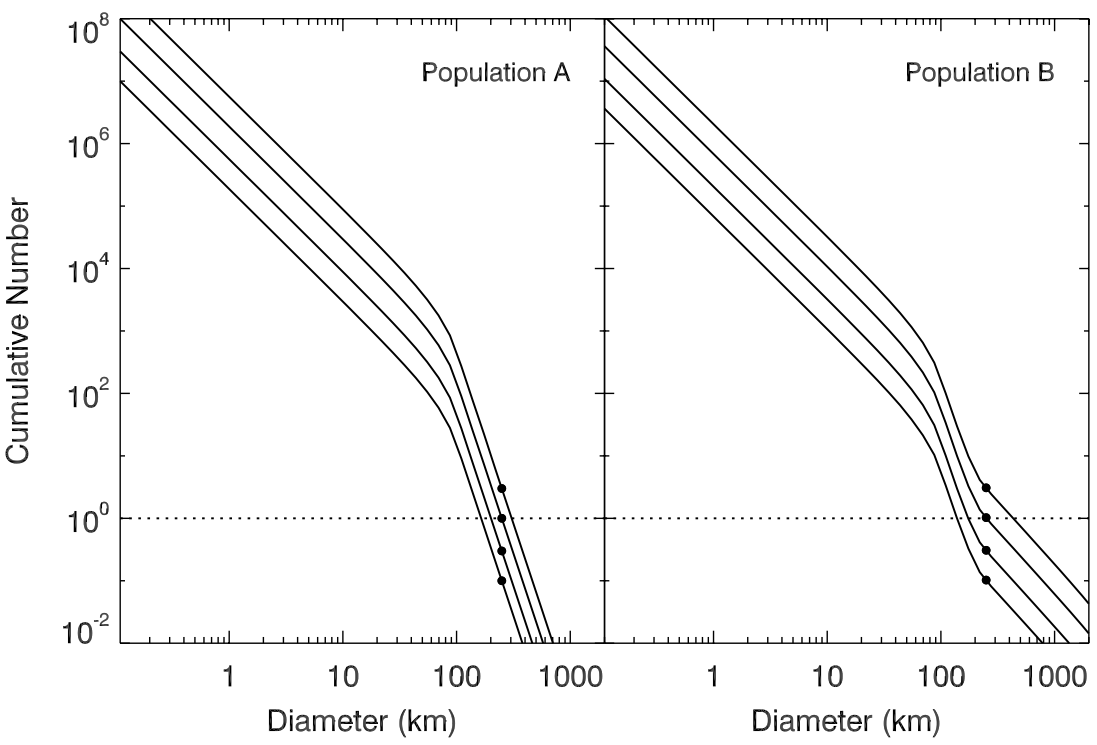

Figure 3. Initial irregular satellite SFDs used to generate our model prograde and retrograde populations. Population A is based on the observed Trojan asteroid SFD with cumulative power-law index $q=-5.5$ for diameter $D>100 \mathrm{~km}$ and $q=-1.8$ for $D<100 \mathrm{~km}$. The populations are normalized by assuming the cumulative number $N(D>250 \mathrm{~km})$ to be $0.1,0.3,1.0$, and $3.0\left(0.0008,0.002,0.008\right.$, and 0.02 lunar masses for $D>0.1 \mathrm{~km}$, respectively, for bulk density $\left.1 \mathrm{~g} \mathrm{~cm}{ }^{-3}\right)$. Population B is the same but assumes there is a shallow branch with $q=-2$ that starts at $D>250 \mathrm{~km}(0.0009,0.003,0.009$, and 0.03 lunar masses for $D>0.1 \mathrm{~km}$, respectively). For reference purposes, we show $N(D>250 \mathrm{~km})=1$ as a dotted line.

assume such impact events create fragments smaller than our resolution limit $(D>0.1 \mathrm{~km})$. Accordingly, we place the total mass of the projectile and target into the code's "trash" bin.

\subsection{Input Parameters for Collision Code}

Here, we describe the parameters needed to run our Boulder simulations. Using the Nesvorný et al. (2007) model as our foundation, we tracked model prograde and retrograde irregular satellite populations captured at Jupiter, Saturn, and Uranus, the planets with sufficient numbers of known satellites to constrain our models, over 3.9 Gyr of collisional evolution (i.e., Neptune is left out). We also assumed that the remnants of the primordial trans-planetary disk passing through these irregular satellite systems were capable of striking and disrupting irregular satellites, though we did not model the collisional evolution of the primordial trans-planetary disk for reasons that will be described below.

Several parameters are needed as input into the code: (1) the starting SFDs of the prograde and retrograde irregular satellites, (2) the SFDs and dynamical decay function for the primordial trans-planetary disk, (3) the collision probabilities and impact velocities between the populations, and (4) the disruption scaling laws and bulk densities for the objects. The code follows the evolution of the irregular satellite SFDs while holding most of these parameters constant. An exception to this is that we forced the remnants of the primordial trans-planetary disk to dynamically decay over time according to the numerical results described in Nesvorný et al. (2007) and Nesvorný \& Vokrouhlický (2009).

The values of these parameters for each of our populations are discussed in the subsections below.

\subsubsection{Initial Size Frequency Distributions of the Irregular Satellites}

The shape of the irregular satellites' SFD immediately after capture is assumed to have the same shape as the Trojan asteroid's SFD. The cumulative number $N(>D)$ of known Trojans (from the "astorb.dat" database described above) can be fit by a broken power law with $q=-5.5$ for $D>100 \mathrm{~km}$ and $q=-1.8$ for $D<100 \mathrm{~km}$, provided the Trojans have a mean albedo of 0.04 (Figure 2).

As a baseline to normalize the Trojan SFD for the irregular satellite populations, we look to the numerical simulations provided by Nesvorný et al. (2007). They predicted that the capture efficiency of irregular satellites from the primordial transplanetary disk to Uranus and Neptune was $(2.7-5.4) \times 10^{-7}$. Thus, if the primordial trans-planetary disk had a mass at $3.9 \mathrm{Ga}$ of $35 M_{\oplus}$, the mass captured at Uranus and Neptune should have been $\sim 0.001$ lunar masses. This is roughly equivalent to the size of the present-day Trojan population, provided the bulk density of the objects was $1 \mathrm{~g} \mathrm{~cm}^{-3}$. This is consistent with the fact that the largest Trojan, (624) Hektor $(D=270 \mathrm{~km}$; Storrs et al. 2005), is only modestly larger than Phoebe $(D=240 \mathrm{~km})$, Himalia $(D=170 \mathrm{~km})$, and Sycorax $(D=150 \mathrm{~km})$ (Figure 1). Nereid at Neptune, which is $D=340 \mathrm{~km}$, is plausible as well.

The source SFDs used to create our model prograde and retrograde satellite SFDs, defined as populations A and B, are shown in Figure 3. The shape of population A is the Trojan SFD described above, namely, a broken power law with $q=-5.5$ for $D>100 \mathrm{~km}$ and $q=-1.8$ for $D<100 \mathrm{~km}$. We assumed our objects had bulk densities of $1 \mathrm{~g} \mathrm{~cm}^{-3}$. The starting mass of the SFD was fixed to values near $0.001-0.01$ lunar masses by assuming the cumulative number $N$ of $D>250 \mathrm{~km}$ objects was $0.1,0.3,1.0$, and 3.0 (nominally $0.0008,0.002,0.008$, and 0.02 lunar masses for $D>0.1 \mathrm{~km}$ ). These values allow us to check whether larger starting populations can be ruled in or out.

Population B mimics population A but has a "foot" attached, namely, a shallow branch with $q=-2$ that starts at $D>$ $250 \mathrm{~km}$. From a probabilistic standpoint, it allows larger objects to be captured into the prograde or retrograde populations. The normalization of population $\mathrm{B}$ is the same as above but the amount of mass captured is slightly higher $(0.0009,0.003,0.009$, and 0.03 lunar masses for $D>0.1 \mathrm{~km}$ ). According to Morbidelli et al. (2009b), the foot might represent objects from the inner component of the primordial trans-planetary disk, which may have been more shallower-sloped than the outer component. 
Table 1

Intrinsic Impact Probability $\left(P_{i}\right)$ of Prograde Irregular Satellites, Retrograde Irregular Satellites, and Remnants from the Primordial Trans-planetary Disk Striking Prograde and Retrograde Irregular Satellite Populations at Jupiter, Saturn, and Uranus

\begin{tabular}{lcccc}
\hline \hline Planet & Pro-Pro & Ret-Ret & Pro-Ret & Disk-Pro/Ret \\
\hline Jupiter & $6.5 \times 10^{-15}$ & $3.8 \times 10^{-15}$ & $1.1 \times 10^{-14}$ & $2.5 \times 10^{-21}$ \\
Saturn & $5.3 \times 10^{-15}$ & $5.4 \times 10^{-15}$ & $1.6 \times 10^{-14}$ & $2.0 \times 10^{-21}$ \\
Uranus & $5.4 \times 10^{-15}$ & $4.6 \times 10^{-15}$ & $1.1 \times 10^{-14}$ & $2.8 \times 10^{-21}$ \\
\hline
\end{tabular}

Note. These numbers are in units of $\mathrm{km}^{-2} \mathrm{yr}^{-1}$.

Observational evidence for a foot may exist in the Kuiper Belt and other populations with large capture/delivery efficiencies (e.g., perhaps the outer main Belt). This issue will be discussed further in the discussion section.

To make our starting conditions as realistic as possible, we also added stochastic elements to mimic the capture process for each trial case of Boulder.

1. The idealized populations $\mathrm{A}$ and $\mathrm{B}$ were divided into $\operatorname{logarithmic}$ intervals $d \log D=0.1$. Random deviates were then used to select objects for the source SFD. In other words, if a given size bin in population A contained 0.1 objects, there would be a $10 \%$ chance that an object of that size would get into the source SFD. This accounts for the fact that the capture of the largest satellites should be probabilistic. Our testbed runs indicate a stochastic element in the capture process could potentially explain the diameter difference between the largest irregular satellites in each system (i.e., Himalia, Phoebe, Sycorax, and Nereid).

2. We defined the parameter $f_{\text {split }}$, the fraction of satellites going into prograde (and retrograde) populations from the source SFD. Using $f_{\text {split }}$ and random deviates, we assigned objects in the source SFD to the prograde or retrograde populations.

For the irregular satellites orbiting Jupiter and Saturn, we tested $f_{\text {split }}=0.4-0.6$, while for those at Uranus, we tested 0.3-0.7 (see below). In the Nesvorný et al. (2007) simulations, captured bodies often had $f_{\text {split }} \approx 0.3-0.5$, but values of $0.6-0.7$ were also found. Our computational work took advantage of the fact that the collision probabilities and impact velocities for prograde-prograde and retrograde-retrograde collisions were similar to one another (see the following section). This means that a trial case for $f_{\text {split }}=0.3$, if the populations are switched, can also be used to examine $f_{\text {split }}=0.7$.

In our production runs, we used the following procedure. (1) We chose a value of $f_{\text {split }} \leqslant 0.5$; we call this $\alpha$. (2) We tested our prograde and retrograde SFDs against the known prograde and retrograde satellites, respectively. (3) We "switched" prograde and retrograde SFDs, which are the equivalent of testing $f_{\text {split }}=1-\alpha$. (4) We ran the same tests as (2).

\subsubsection{Collision Probabilities and Impact Velocities}

Immediately after capture at their primary planets, the prograde and retrograde irregular satellite populations are capable of striking both themselves and each other. Using this idea, we took the $(a, e, i)$ values of the test bodies captured onto stable orbits within the Nesvorný et al. (2007) simulations and computed collision probabilities and impact velocities for each population using the code described in Bottke et al. (1994). Our output parameters were $P_{i}$, the average "intrinsic collision probability" (i.e., the probability that a single member of the impacting population will hit a unit area of a body in the target population over
Table 2

Impact Velocity ( $V_{\mathrm{imp}}$ ) in Units of $\mathrm{km} \mathrm{s}^{-1}$ of Prograde Irregular Satellites, Retrograde Irregular Satellites, and Remnants from the Primordial Trans-planetary Disk Striking Prograde and Retrograde Irregular Satellite Populations at Jupiter, Saturn, and Uranus

\begin{tabular}{lcccc}
\hline \hline Planet & Pro-Pro & Ret-Ret & Pro-Ret & Disk-Pro/Ret \\
\hline Jupiter & 3.1 & 3.1 & 6.7 & 7.0 \\
Saturn & 1.4 & 1.4 & 4.0 & 4.7 \\
Uranus & 1.0 & 1.0 & 2.1 & 3.0 \\
\hline
\end{tabular}

a unit of time), and the mean impact velocity $V_{\text {imp. These values }}$ are described in Tables 1 and 2.

The test bodies used here were taken from Run 9 of Nesvorný et al. (2007). At Jupiter, the numbers of test bodies found on stable prograde and retrograde orbits were 153 and 188, respectively. At Saturn, the values were 218 and 219, respectively, while for Uranus, the numbers were 880 and 918, respectively. Neptune was not examined (see Section 2). Using these objects, we found $P_{i}$ values ranging from $3.8 \times 10^{-15} \mathrm{~km}^{-2} \mathrm{yr}^{-1}$ to $1.6 \times 10^{-14} \mathrm{~km}^{-2} \mathrm{yr}^{-1}$. Values calculated from the real irregular satellites produced comparable results.

These values are remarkably high compared to the values we are used to seeing for small body populations. To put this into context, consider the following. The starting population for the irregular satellites was 50 times less populous than the main asteroid Belt population (0.001 lunar masses versus 0.05 lunar masses, respectively). The $P_{i}$ values for the irregular satellites, however, are 1000-6000 times higher than typical main Belt values ( $P_{i}=2.85 \times 10^{-18} \mathrm{~km}^{-2} \mathrm{yr}^{-1}$; Bottke et al. 1994), while their impact velocities, which range from $1 \mathrm{~km} \mathrm{~s}^{-1}$ to $7 \mathrm{~km} \mathrm{~s}^{-1}$ are comparable or only modestly lower than typical main Belt values $\left(V_{\mathrm{imp}}=5.3 \mathrm{~km} \mathrm{~s}^{-1}\right.$; Bottke et al. 1994). Put together, we find the irregular satellites immediately after capture should act like an asteroid Belt containing 20-100 times more mass than it has today (Bottke et al. 2005a, 2005b).

The take-away message from these parameters is that even populations of modest masses trapped around the gas giants must undergo an enormous degree of collisional evolution, perhaps more than any other surviving small body population has yet experienced.

\subsubsection{The Primordial Trans-planetary Disk Population}

The other population that can collide with the irregular satellite SFDs is the surviving remnant of the primordial transplanetary disk. This population readily decays as the gas giant scatters away its members, but not so quickly that it can be ignored. For added realism, we included it in our model.

Figure 4 shows two estimates for this population just prior to Jupiter and Saturn crossing into a mutual mean motion resonance, one containing $\approx 20 M_{\oplus}$ and one with $\approx 40 M_{\oplus}$ (e.g., Tsiganis et al. 2005). As before, we assume that at the moment the instability started, the disk SFD had the same shape as the current Trojan SFD. These populations were normalized by assuming there were $7.5 \times 10^{5}$ and $1.5 \times 10^{6}$ objects with $D>200 \mathrm{~km}$, respectively. Note that most of the mass in each SFD is near the inflection point at $D=100 \mathrm{~km}$, such that the large body end of the SFD does not play a meaningful factor in the evolution of the irregular satellites.

To include this population in Boulder, we must account for the fact that the primordial trans-planetary disk is scattered and cleared during the Nice model simulations. This means that the number capable of hitting the irregular satellites decreases 


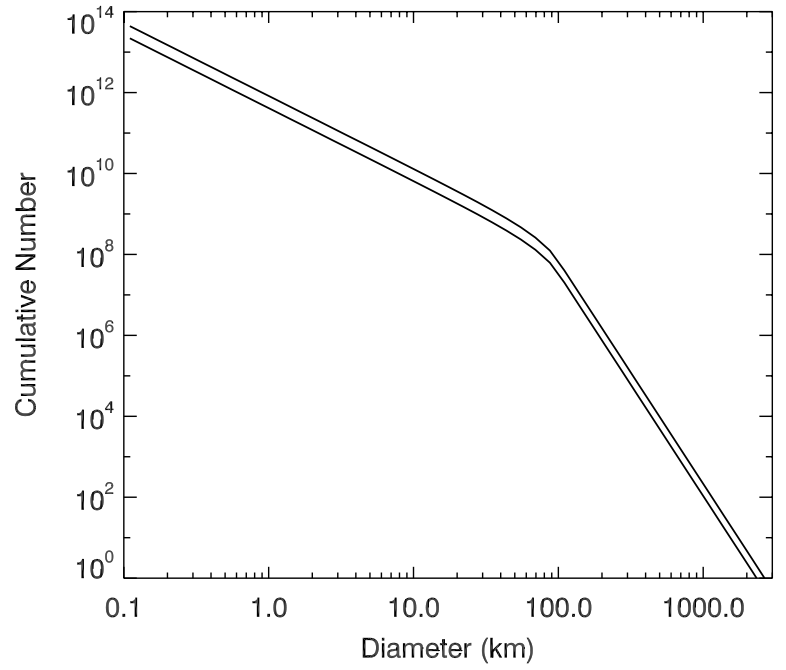

Figure 4. Estimates of the SFD of the primordial trans-planetary disk just prior to the capture of the irregular satellites by the gas giants. Some of these objects go on to strike captured irregular satellites. We assume the population had the same shape as population A (Figure 2); cumulative power-law index $q=-5.5$ for diameter $D>100 \mathrm{~km}$ and $q=-1.8$ for $D<100 \mathrm{~km}$. The total masses of the systems are $20.4 M_{\oplus}$ and $40.8 M_{\oplus}$ for diameter $D>0.1 \mathrm{~km}$ objects. These two populations were normalized by assuming that there are $7.5 \times 10^{5}$ and $1.5 \times 10^{6}$ objects with $D>200 \mathrm{~km}$, respectively.

dramatically with time. At the same time, we also need to couple this information to the $P_{i}$ and $V_{\text {imp }}$ values computed between the remnant disk population and the irregular satellite populations.

We characterized the scattering of the disk using new runs based on the Nice model template described in Nesvorný et al. (2007), Nesvorný \& Vokrouhlický (2009), and in Section 3.1. Here we started 27,000 disk particles (rather than 7000) and integrated them out to $1 \mathrm{Gyr}$ (as opposed to $130 \mathrm{Myr}$ ). Particles were removed from the system if they struck a planet, the Sun, or were thrown out of the outer solar system. For computational expediency, we assumed the same decay curve for objects crossing the orbits of Jupiter and Saturn.

Next, using these data and the techniques described in Charnoz et al. (2009), we computed $P_{i}$ and $V_{\text {imp }}$ values between disk particles passing near the gas giants and the irregular satellite populations. A running mean of these values was used to eliminate jitter. In some cases, we also interpolated through the values when it was clear that it could be fit to a line segment (e.g., see Bottke et al. 2005b). Beyond $600 \mathrm{Myr}$, we assumed that the remnant populations shown in the figure were a reasonable "stand-in" for the refugees from the Kuiper Belt and scattered disk that occasionally hit the irregular satellites. Accordingly, we assumed that the impacting disk population did not change in a meaningful way beyond this time.

Our results, which are shown in Figure 5, show that the primordial trans-planetary disk population decays rapidly, with a slower drop off for objects near Uranus than those near Jupiter and Saturn. This occurs because Uranus (and Neptune) are embedded in the disk while Jupiter and Saturn are effectively decoupled from the population. Test results also indicate that the decay is fast enough that the irregular satellites are only seriously affected by disk-satellite collisions during two intervals: the first few tens of Myr of the simulation, where the population is still large, and last several Gyr of the simulation, where the irregular satellite populations have become so decimated that the sporadic impacts of disk bodies actually have some effect.

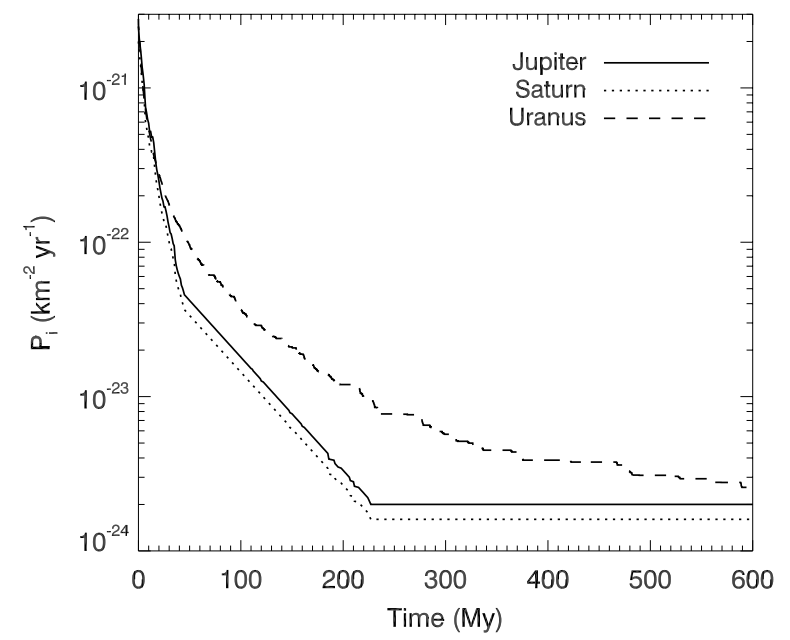

Figure 5. Mean intrinsic collision probabilities between individual objects in the primordial trans-planetary disk (see Figure 3 ) and the irregular satellites at Jupiter, Saturn, and Uranus as a function of time. These values are based on test body simulations of the dynamical scattering of the disk. A running mean of our values is plotted to eliminate jitter in the plot. We also use interpolated values when the data could be fit to a line segment. This explains the flat line for Jupiter and Saturn at $P_{i} \approx 2 \times 10^{-24} \mathrm{~km}^{-2} \mathrm{yr}^{-1}$. We assumed that after $600 \mathrm{Myr}$, the remnants of the impacting disk population, which we assumed were a proxy for the ecliptic comets that had escaped the Kuiper Belt and scattered disk populations, did not change.

Finally, we find that the impact velocities $V_{\text {imp }}$ between the remnant disk population and the irregular satellite populations do not change very much throughout the simulation. The values used for impacts on the irregular satellites at Jupiter, Saturn, and Uranus are $7.0 \mathrm{~km} \mathrm{~s}^{-1}, 4.7 \mathrm{~km} \mathrm{~s}^{-1}$, and $3.0 \mathrm{~km} \mathrm{~s}^{-1}$, respectively (Table 2). The higher impact velocities found for impacts at Jupiter and Saturn compensate somewhat for their more rapid decay curves (Figure 5).

\subsubsection{Disruption Scaling Laws and Bulk Densities for Irregular Satellites}

For a $Q_{D}^{*}$ function applicable to irregular satellites, we turn to Levison et al. (2009), who recently used Boulder to model the collisional evolution of icy planetesimals in the primordial transplanetary disk that were scattered into the outer main Belt, Hilda, and Trojan asteroid populations. If the irregular satellites came from that same source population, it is reasonable to assume that the irregular satellites have the same disruption properties as the C-, D-, and P-type objects modeled in Levison et al. (2009).

In Levison et al. (2009), the $Q_{D}^{*}$ function was assumed to split the difference between SPH impact experiments of Benz \& Asphaug (1999), who used a strong formulation for ice, and those of Leinhardt \& Stewart (2009), who used the finite volume shock physics code CTH to perform simulations into what they describe as weak ice. To do this, Levison et al. (2009) examined what happened when the Benz \& Asphaug (1999) strong ice $Q_{D}^{*}$ function was divided by a factor, $f_{Q}$. They found that the best match to the observed populations came from using $f_{Q}=3$, 5 , and 8 (see Figure 6). Note that because we sampled a broad section of parameter space, we chose not to include still more complicating factors (e.g., $Q_{D}^{*}$ varies with impact velocity, etc.).

The bulk densities $(\rho)$ of typical irregular satellites are largely unknown; direct estimates are only available for the largest Jovian and Saturnian satellites. Phoebe, which the Cassini spacecraft encountered in 2004, has $\rho=1.634 \mathrm{~g}$ $\mathrm{cm}^{-3}$ (Jacobson et al. 2006). Himalia, which has produced 


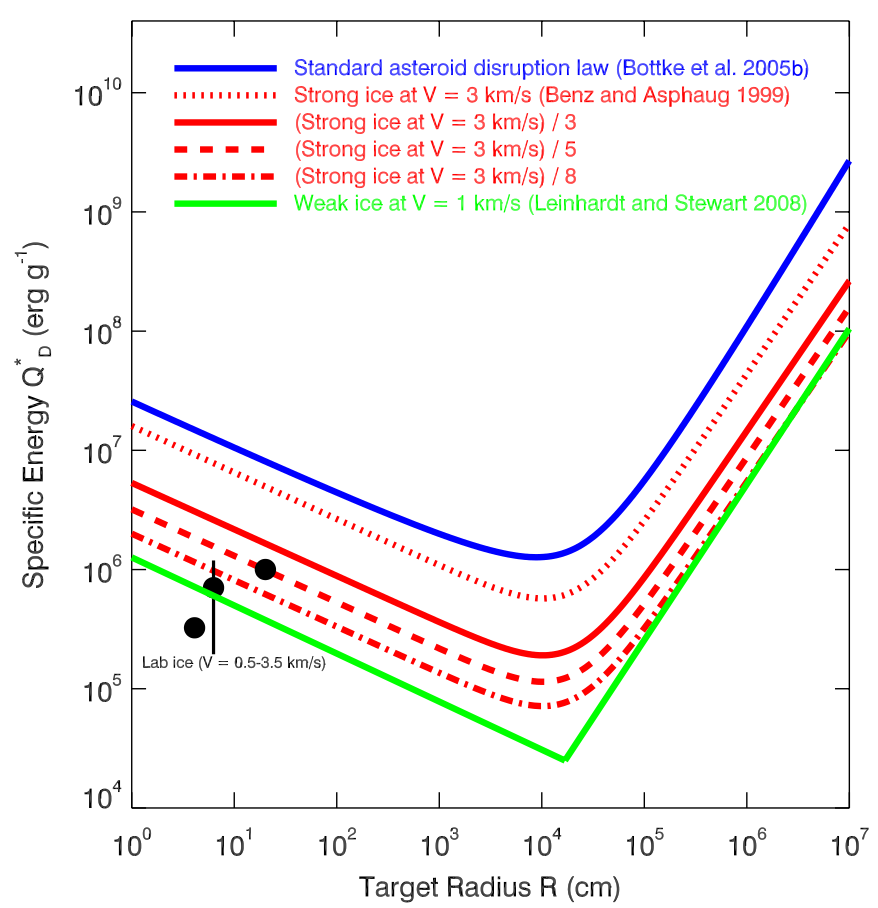

Figure 6. Disruption scaling law $Q_{D}^{*}$ functions described in the text. We define the function $Q_{D}^{*}$, the critical specific impact energy, as the energy per unit target mass needed to disrupt the target and eject $50 \%$ of its mass. The blue curve shows the standard disruption function for asteroids derived by Bottke et al. (2005b). The green curve shows the minimum value for weak ice as determined from numerical CTH impact experiments, while the black dots are data from laboratory disruption experiments into weak nonporous ice (Leinhardt \& Stewart 2009). The dotted red curve is data from numerical SPH impact experiments on ice targets using a strong formulation for ice (Benz \& Asphaug 1999). This is our standard $f_{Q}=1$ function. Finally, the solid, dashed, and dot-dashed red curves show $f_{Q}=3,5$, and 8 , respectively.

measurable gravitational perturbations on nearby satellite Elara, appears to have a comparable or perhaps much higher $\rho$ value, though much depends on its exact shape and size (Emelyanov 2005).

By assuming the irregular satellites came from the primordial trans-planetary Belt, however, we can assume that these objects have comparable $\rho$ values to other objects that came from the disk, namely comets, Kuiper Belt objects, and Trojan asteroids. A survey of the literature produces $\rho$ values of between 0.46 (Centaur $2002 \mathrm{CR}_{46}$; Noll et al. 2006) and 2.5 $\mathrm{g} \mathrm{cm}^{-3}$ (Binary Trojan (624) Hektor; Lacerda \& Jewitt 2007; see also a recent review by Weissman et al. 2004). Taken together, we choose to make the same assumption made by Levison et al. (2009), namely we will give our objects cometlike bulk densities of $1 \mathrm{~g} \mathrm{~cm}^{-3}$. This $\rho$ value was selected to roughly split the difference between the extremes of the above values.

\subsubsection{Caveats}

While we consider our Boulder runs to be state of the art, they are still essentially one dimensional (i.e., we assume individual $P_{i}, V_{\text {imp }}$ values and single SFDs represent all the components within different irregular satellite sub-populations). In reality, one can find many examples of prograde objects that do not cross retrograde ones or zones near large irregular satellites that are essentially clear of debris (see the discussion in Nesvorný et al. 2003). It is important to start somewhere, though, and comparable models have yielded useful insights into the origin and evolution of the asteroid Belt (e.g., Davis et al. 2002; Bottke et al. 2005a, 2005b). Moreover, the insights gleaned from our runs will help us develop more sophisticated models in the future.

\section{RESULTS}

In our production runs, we tested how different prograde and retrograde irregular satellite populations at Jupiter, Saturn, and Uranus were affected by 4 Gyr of collisional evolution. We assumed that the dynamical capture and collisional evolution of these populations were stochastic in nature. Accordingly, for each set of starting conditions, we executed 50 trial cases and output our results every $1 \mathrm{Myr}$ over the $4 \mathrm{Gyr}$ evolution time. We define this body of work as an individual "run."

Each run is defined by five parameters: the shapes of the irregular satellite SFDs (i.e., populations A and B, which are defined using $N(D>250 \mathrm{~km})=0.1,0.3,1.0$, and 3.0, and $q=-2$ and -5.5 for $D>250 \mathrm{~km})$, the fraction of objects that go into prograde (and retrograde) orbits around the primary planet $\left(f_{\text {split }}=0.3,0.4,0.5\right.$; see Section 3.3.1), the masses of the primordial trans-planetary disk at the time Jupiter and Saturn entered into a mutual mean motion resonance (i.e., 20.4 and $40.8 M_{\oplus}$ ), and the disruption scaling laws for the irregular satellites, which we assumed were comet-like bodies (i.e., $f_{Q}=1,3,5$, and 8). All told, we performed 384 production runs $(19,200$ trial cases) and created 76.8 million output SFDs to model the irregular satellites populations at Jupiter, Saturn, and Uranus. We also performed additional runs to rule our parameter combinations that predominantly produce unsatisfying fits (e.g., $f_{\text {split }}=0.5$ for Uranus). These will be described below as needed.

The amount of data generated in our runs meant we could not examine all of it by eye. We dealt with this by developing an automatic scoring and analysis routine to tell us when the output model SFDs from our trial cases fit the observational data beyond some threshold value. Our scoring procedure is described in the following section.

\subsection{Scoring the Results}

One of the most complex issues in this project is determining when a good match exists between our parameter-dependent model SFDs and the observed irregular satellite SFDs. While there is a strong temptation to compare both "by eye" and simply report the results, this becomes impractical when we are dealing with hundreds of runs that contain millions of output SFDs. Moreover, the results would be highly subjective and would differ between observers.

For this reason, we decided that it would be useful to introduce a scoring (target) function that helps us define the comparison of the modeled and observed SFDs quantitatively. In our first attempts, we tried to apply statistical chi-square-like tests (e.g., Press et al. 1992) to the data. Unfortunately, we found them difficult to apply in our situation because (1) the observed SFDs had poorly defined uncertainties and arbitrary bin sizes, (2) the model SFDs output from Boulder were assigned ever-changing bin center locations as collisional evolution took place, and (3) the uncertainties in the sizes of the observed objects were, in most cases, estimated from an albedo assumption rather than by a rigorous method.

In this situation, we decided to use a much simpler tool where the quantitative threshold of "quality" in the model-todata comparison was obtained using a predefined metric rather than an exact statistical method. The advantage to this method 
was that it was fast, easy to evaluate, and did not directly use the unreliable uncertainties in the satellite sizes. The disadvantage is that we cannot assign a statistical level of confidence to our results. With that cautionary note, we describe our method below.

We assume that $D_{i}^{(O)}, i=1, \ldots, N$, defines a vector of sizes for the observed population of irregular satellites, while $D_{i}^{(C)}$ is the same for the modeled population. No bin has more than one member; if the modeled population has a size bin occupied by several objects, we spread out the respective number of copies in $D_{i}^{(C)}$. The quality of the match between the observed and modeled populations is then defined as the scoring function:

$$
S=\frac{1}{N} \sum_{i=1}^{N}\left|\log \left(\frac{D_{i}^{(C)}}{D_{i}^{(O)}}\right)\right| .
$$

The $S$-score is evaluated separately for both the prograde and retrograde irregular satellite populations. The modeled population typically contains many more objects than the observed one, $N$ in our case, and we consider the first $N$ largest bodies in the modeled population to evaluate the score function (3). In some cases, though, one of the irregular populations might become decimated by the other, such that the modeled population could contain fewer objects than the observed one. In that case, $N$ in Equation (3) becomes the number of modeled objects. Note that if $N$ ever equals zero, $S$ is assigned an arbitrarily large value indicating a poor match.

As an example of how to use $S$, consider a modeled SFD whose largest objects get within a factor of 2 in diameter to the members of the observed SFD. In that circumstance, $S$ drops to a value of 0.3 or smaller. Given all other uncertainties and biases, we consider this level of agreement to be reasonable enough that we will assume $S \simeq 0.3$ is the acceptable threshold of a "good" match. It also produces results that are consistent with our "by eye" evaluations.

\subsection{Jupiter's Irregular Satellites}

We start our description of our model results by showing off a successful trial case. Figure 7 shows eight snapshots from a trial case for Jupiter's irregular satellites with input parameters $f_{Q}=3, f_{\text {split }}=0.4, N(D>250 \mathrm{~km})=0.1, q=-5.5$, and $M_{\text {disk }}=40 M_{\oplus}$. The initial conditions are shown in the time $t=0$ Myr panel (top left). Here $f_{\text {split }}$ specifically corresponds to $40 \%$ of the population going into the prograde population. The model retrograde and prograde SFDs are represented by green and magenta dots, respectively, while those of the observed retrograde and prograde SFDs are given by the blue and red dots, respectively.

The top end of the model SFDs shows off the stochastic nature of the capture process. For example, for $f_{\text {split }}=0.4$, the model prograde objects ended up with $1 D=180 \mathrm{~km}$ object, $4 D=$ $110 \mathrm{~km}$ objects, and $11 \mathrm{D}=90 \mathrm{~km}$ objects, while the retrograde object obtained $2 D=140 \mathrm{~km}$ objects, $6 \mathrm{D}=110 \mathrm{~km}$ objects, and $18 D=90 \mathrm{~km}$ objects. Thus, while the fraction of objects on prograde versus retrograde orbits is what we would expect (0.38), the mass distribution can be a variable.

The first two time steps, $t=1 \mathrm{Myr}$ and $10 \mathrm{Myr}$, show that the two populations grind away fastest when they are largest. For example, at $10 \mathrm{Myr}$, each SFD has decreased by factors of 3-10 over nearly their entire span. We find the shape of the SFDs for $D<10 \mathrm{~km}$ largely depends on the nature of the breakup events occurring at larger sizes. Particularly large super-catastrophic

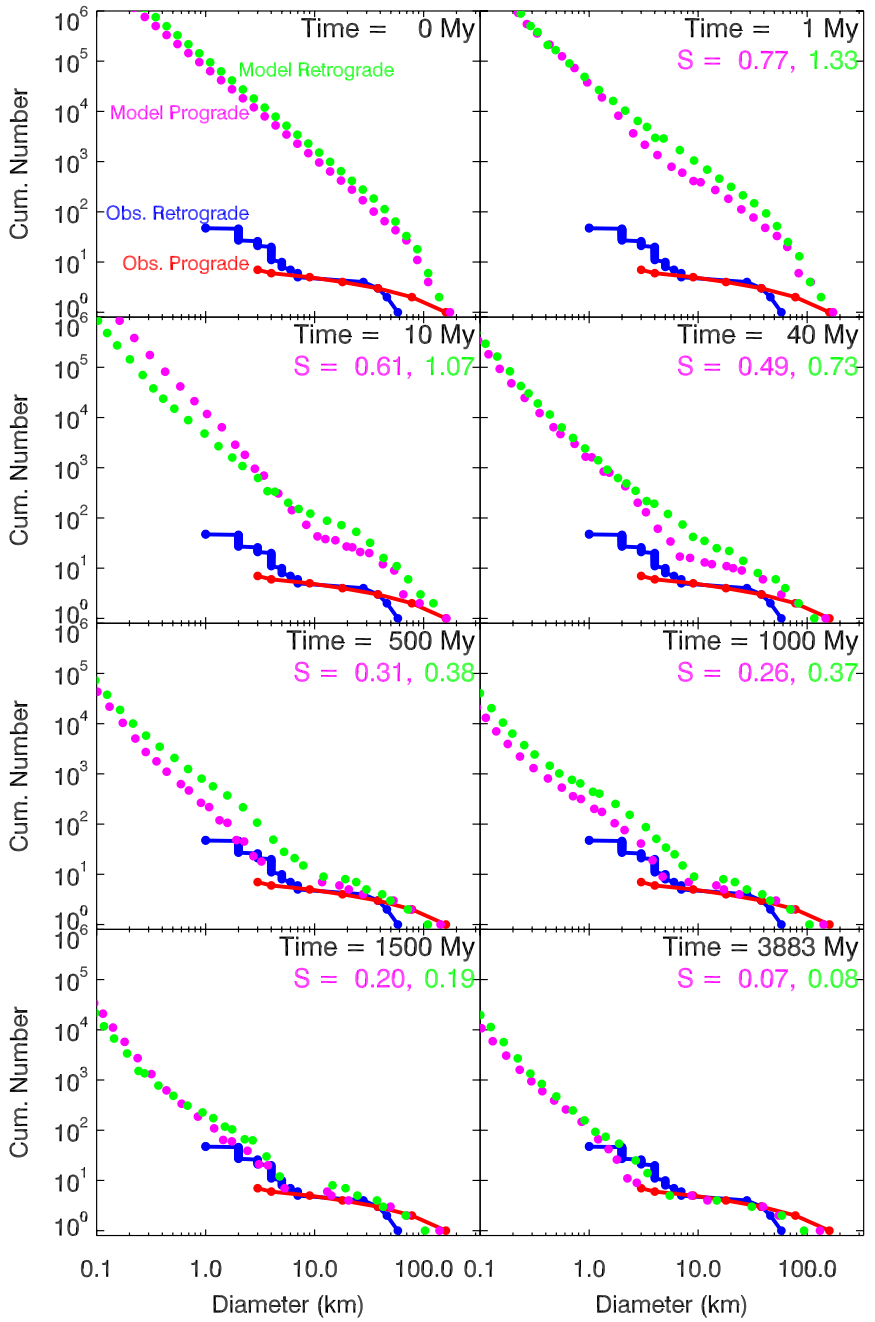

Figure 7. Collisional evolution of Jupiter's irregular satellites. We assumed input parameters $f_{Q}=3, f_{\text {split }}=0.4, N(D>250 \mathrm{~km})=0.1, q=-5.5$, and $M_{\text {disk }}=40 M_{\oplus}$. Our initial conditions are shown in the time $t=0 \mathrm{Myr}$ panel (top left). The model retrograde and prograde SFDs are represented by green and magenta dots, respectively, while those of the observed retrograde and prograde SFDs are given by the blue and red dots, respectively. The score $S$ of the prograde and retrograde population at each snapshot time is shown in the legend. Our runs show the populations quickly grind away, enough that they approach their end-state steady-state condition somewhere between 40 and 500 Myr. The last two frames show $S<0.3$ for both populations, values we consider good fits. Note that the fragment tail for diameter $D<8 \mathrm{~km}$ wags up and down in response to large catastrophic disruption or cratering events, though this is not shown in the individual frames. The last frame shows our best-fit time, where excellent fits are achieved except for the largest objects in each population.

disruption events often dominate the population at smaller sizes for extended periods. This allows the prograde and retrograde SFDs for $D<5 \mathrm{~km}$ to undergo sudden and sometimes radical changes. In fact, it is common to see the SFDs jump to steeper slopes in the aftermath of a large collision event and then slowly retreat to shallow slopes as widespread grinding beats the new fragment population back down. Both prograde and retrograde scores $S$ for $1-10 \mathrm{Myr}$ are $>0.5$, indicating that we are still far from a satisfying match to the observational data. At $t=40 \mathrm{Myr}$, however, the SFDs begin to take on shapes that are similar, in many ways, to the observed populations.

The endgame of the satellite evolution simulation begins near $t=500$ Myr. Here the matches between model and observations approach $S \approx 0.3$, the minimum threshold value needed for a good fit. At this point, both populations are beaten 
Table 3

Top Five Trial Cases from Our Irregular Satellite Runs for Jupiter, Saturn, and Uranus

\begin{tabular}{lcccccc}
\hline \hline Planet & $f_{Q}$ & $f_{\text {split }}$ & $N(D>250 \mathrm{~km})$ & $q$ & $M_{\text {disk }}\left(M_{\oplus}\right)$ & Frac. Success \\
\hline Jupiter & 3 & 0.5 & 1.0 & -5.5 & 40.8 & $0.55 \pm 0.056$ \\
Jupiter & 3 & 0.4 & 0.1 & -5.5 & 20.4 & $0.50 \pm 0.058$ \\
Jupiter & 3 & 0.4 & 0.1 & -2.0 & 20.4 & $0.47 \pm 0.059$ \\
Jupiter & 3 & 0.4 & 0.1 & -5.5 & 40.8 & $0.45 \pm 0.057$ \\
Jupiter & 3 & 0.5 & 0.1 & -5.5 & 40.8 & $0.43 \pm 0.057$ \\
Saturn & 3 & 0.5 & 3.0 & -5.5 & 40.8 & $0.34 \pm 0.050$ \\
Saturn & 3 & 0.4 & 0.1 & -5.5 & 40.8 & $0.32 \pm 0.048$ \\
Saturn & 1 & 0.5 & 0.1 & -2.0 & 40.8 & $0.32 \pm 0.057$ \\
Saturn & 5 & 0.5 & 1.0 & -5.5 & 20.4 & $0.31 \pm 0.050$ \\
Saturn & 1 & 0.4 & 0.1 & -5.5 & 40.8 & $0.31 \pm 0.053$ \\
Uranus & 8 & 0.3 & 1.0 & -5.5 & 20.4 & $0.25 \pm 0.060$ \\
Uranus & 5 & 0.3 & 1.0 & -5.5 & 20.4 & $0.23 \pm 0.058$ \\
Uranus & 3 & 0.3 & 1.0 & -5.5 & 20.4 & $0.17 \pm 0.054$ \\
Uranus & 8 & 0.3 & 3.0 & -5.5 & 40.8 & $0.16 \pm 0.052$ \\
Uranus & 8 & 0.3 & 0.3 & -5.5 & 20.4 & $0.16 \pm 0.050$
\end{tabular}

Notes. We selected the top five using the following criteria. We compared the model SFDs in every trial case to the observed SFDs every 1 Myr over the last $0.5 \mathrm{Gyr}$ of our $4 \mathrm{Gyr}$ simulations. Using the score $S$ parameter described in Section 4.1, we tracked how many times the prograde and retrograde populations both had $S<0.3$. The top five were those trial cases with the highest success values as shown in the fractional success column. The remaining columns are the initial parameters used to generate those trial cases. Planet corresponds to the central planet of the irregular satellites in question. The value $f_{Q}$ corresponds to the disruption scaling law used for the trial case (i.e., we assume the specific critical energy density $Q_{D}^{*}$ needed to catastrophically disrupt a target made of strong ice as defined by Benz \& Asphaug (1999) was divided by a factor $f_{Q}$ ). The fraction of satellites started in the prograde population is $f_{\text {split }}$. Our initial SFDs are normalized to the value $N(D>250 \mathrm{~km})$ and have a cumulative power-law index for $D>250 \mathrm{~km}$ objects of $q$. Finally, the total mass in the primordial trans-planetary disk at the time the irregular satellites were captured was $M_{\text {disk }}$ in units of $M_{\oplus}$.

up and battered. The large body populations have become so depleted that, at any given time, most small fragments are produced by cratering events. Sporadic large-scale catastrophic disruption events, however, can and do dominate the small body populations from time to time. This means the shapes of the SFDs for $D<5 \mathrm{~km}$ wiggle and wag up and down for the next several billion years. Occasionally, the match with the observed SFD becomes quite good, as seen for $t=3883 \mathrm{Myr}$. This cannot last, though, and collisional evolution over several tens of $\mathrm{Myr}$ is enough to degrade the fit until the next big disruption event restarts the process. In other words, all of this has happened before and will happen again.

Table 3 shows the parameter sets for the top five runs that produced good fits $(S<0.3)$ to the observed prograde and retrograde over the last $500 \mathrm{Myr}$. These parameters produce good fits with the data approximately $50 \%$ of the time over the tested interval. We consider this remarkable when one considers all of the possible ways our simulations could have run into trouble. The common theme in the top five runs appears to be $f_{Q}=3$ and an input SFD without a foot $(q=-5.5)$ that was normalized for $N(D>250 \mathrm{~km})=0.1$. To some degree, this would argue against input SFDs that contain more large bodies.

We found that using the top five runs alone to analyze trends across 128 runs was generally an unsatisfying way to sift our data. For that reason, we decided to augment our analysis by combining our results across all runs. Here we computed the average number of good fits found over the last $500 \mathrm{Myr}$ in each run and combined these data across our five input parameters. Our net results are shown in Figures 8 and 9.

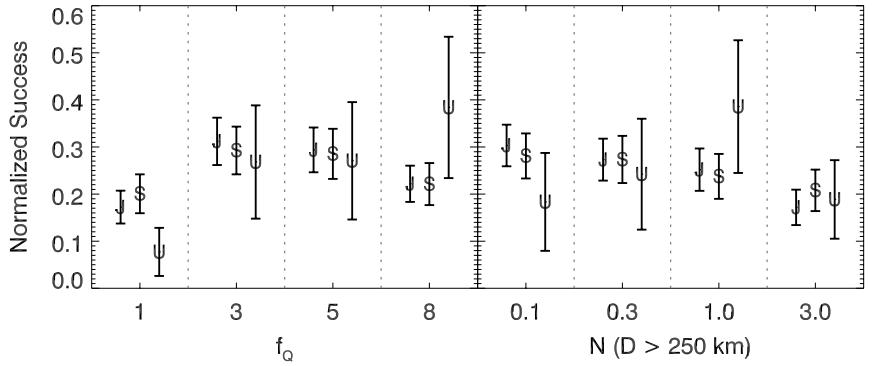

Figure 8. Input parameter preferences for $f_{Q}$ and $N(D>250 \mathrm{~km})$. We computed the number of good fits $(S<0.3$ for the prograde and retrograde populations) over the last $0.5 \mathrm{Gyr}$ of our $4 \mathrm{Gyr}$ simulations for each trial case. Next, we averaged these values for each run. We sifted these values according to the input parameter value and summed them. We then normalized these values to 1.0 and plotted then against one another. The result is shown in the plot. The letters $\mathrm{J}, \mathrm{S}$, and $\mathrm{U}$ stand for the irregular satellite values obtained from our irregular satellite runs at Jupiter, Saturn, and Uranus, respectively. Here we show our model's preferences for $f_{Q}$, which was given values of $1,3,5$, and 8 , and $N(D>250 \mathrm{~km})=0.1,0.3,1.0$, and 3.0. The trends across all planets indicate that $f_{Q}>3$ and $N(D>250 \mathrm{~km})<3.0$ are most likely to produce good scores between model and data. The value $f_{Q}=8$ and $N(D>250 \mathrm{~km})=1.0$ are particularly favored for Uranus.

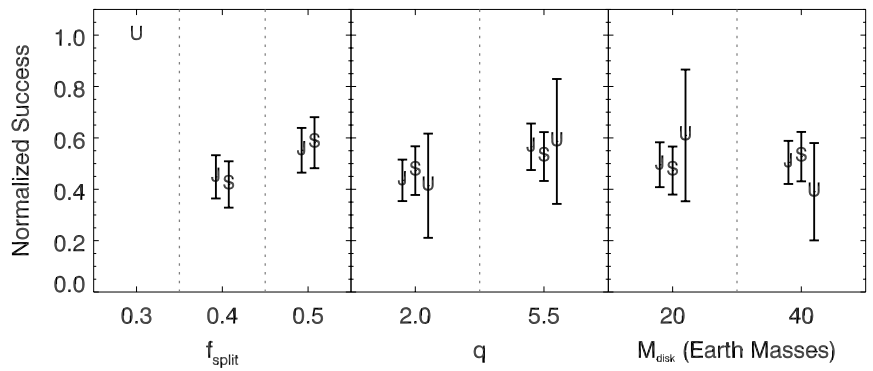

Figure 9. Input parameter preferences for $f_{\text {split }}, q$, and $M_{\text {disk }}$. Our procedure and labels are explained in Figure 8. Here we examine $f_{\text {split }}=0.4$ and 0.5 for our Jupiter and Saturn runs, $q$ values of -2.0 and -5.5 for all planets, and $M_{\text {disk }}$ for 20 and $40 M_{\oplus}$. The trends are modest, but $f_{\text {split }}$ values of 0.5 and $q$ values of -5.5 are favored. There does not appear to be any trend for $M_{\text {disk }}$.

In Figure 8, our Jupiter runs show preferences for $f_{Q} \geqslant 3$, with 3 being the favorite, and for smaller values of $N(D>$ $250 \mathrm{~km}$ ), with 0.1 being the favorite. In Figure 9, the trends are less distinct, but $f_{\text {split values of } 0.5 \text { and } q \text { values of }-5.5}$ are generally favored. There does not appear to be any trend for $M_{\text {disk. }}$. These values are in generally good agreement with the trends seen for the top five runs from Table 3, giving us confidence that both approaches yield useful results.

\subsection{Saturn's Irregular Satellites}

Saturn's irregular satellites were tested against the same parameter suite as that used for Jupiter's runs. Thus, in terms of collisional evolution, the only model differences between the satellite systems should be Saturn's $P_{i}$ values, which vary slightly from Jupiter's (Table 1), and Saturn's significantly lower

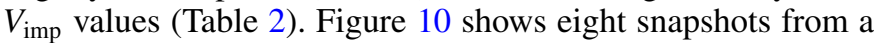
trial case for Saturn's irregular satellites. Our initial conditions are shown in the time $t=0 \mathrm{Myr}$ panel. The color code for the different populations is the same as in Figure 7.

To demonstrate that there are many ways to get reasonable fits, we show a trial case here with somewhat different parameters than Figure 7: $f_{Q}=8, f_{\text {split }}=0.6, N(D>250 \mathrm{~km})=0.3$, $q=-5.5$, and $M_{\text {disk }}=40 M_{\oplus}$. Here an important difference from Figure 7 is that $60 \%$ of the starting population goes into the model prograde population rather than the model retrograde one. A larger starting prograde population can be useful because the 


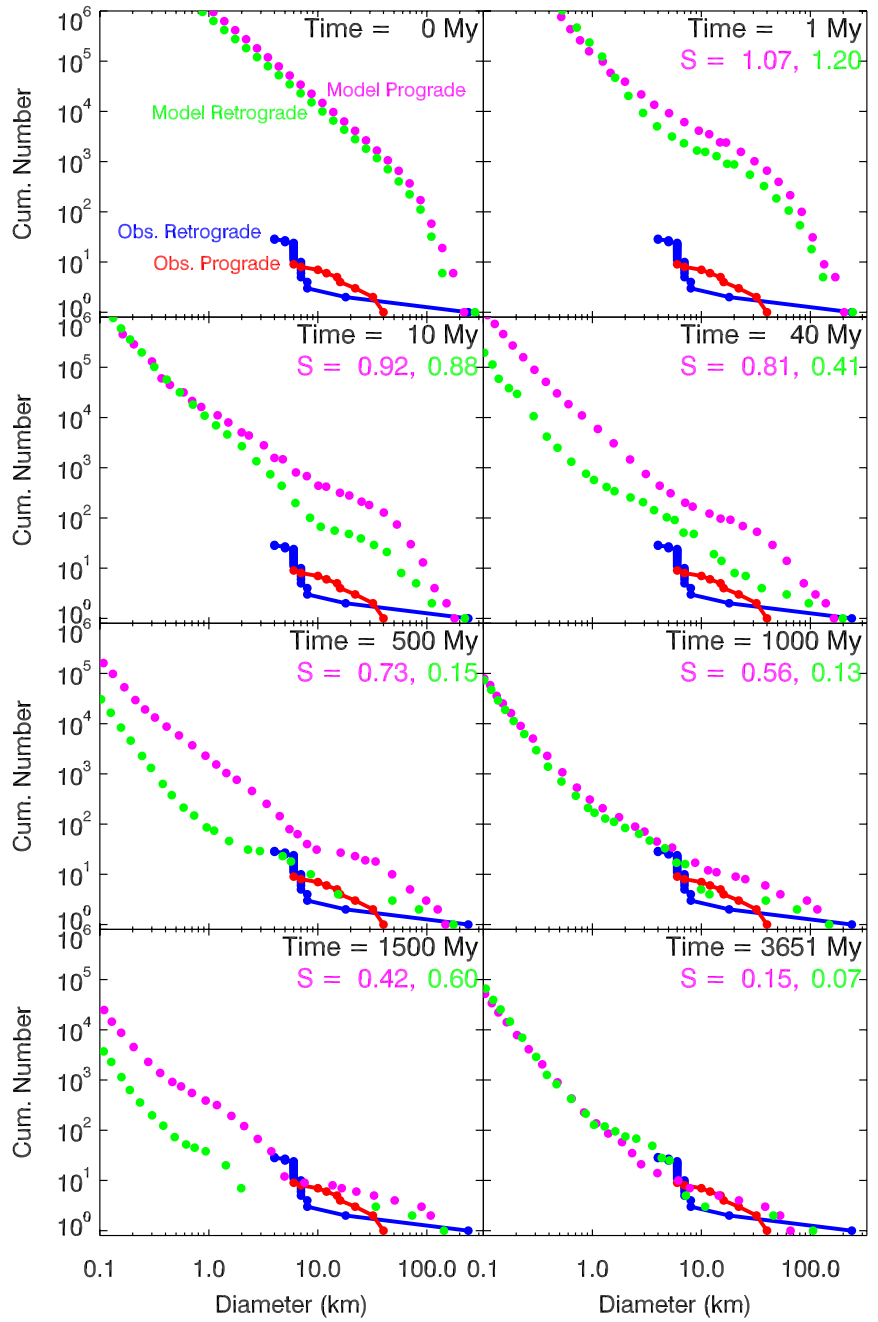

Figure 10. Collisional evolution of Saturn's irregular satellites. The color code and labels are the same as in Figure 7. The input parameters used here were $f_{Q}=8, f_{\text {split }}=0.4$ (though here it corresponds to the retrograde population; this is equivalent because the collisional probabilities and impact velocities are symmetric $), N(D>250 \mathrm{~km})=0.3, q=-5.5$, and $M_{\text {disk }}=40 M_{\oplus}$. Our initial conditions are shown in the time $t=0 \mathrm{Myr}$ panel (top left). The objects grind away in much the same fashion as in Figure 7, with most of the mass depleted $500 \mathrm{Myr}$ into the simulation. The large end of the size distribution, however, requires billions of years of comminution to reach the best-fit time at 3651 Myr. Note that our largest model retrograde object is a factor of 2 smaller than Phoebe. This is a common problem; we have yet to find a trial run using our input parameters that produces the observed gap in size between Phoebe and the second largest retrograde satellite. This could suggest that Phoebe is special in some manner (e.g., it was captured by a different dynamical mechanism) or that something interesting has happened to the Saturnian system.

observed prograde satellites have more $D=10-30 \mathrm{~km}$ bodies than the observed retrograde bodies.

While the shapes of the SFDs through the first $500 \mathrm{Myr}$ in Figure 10 are reminiscent of those seen in Figure 7, the factor of 2 decrease in $V_{\text {imp }}$ values at Saturn when compared to Jupiter mean typical collisions are less energetic. The consequence is that the SFDs grind away more slowly, which helps explain the larger abundance of $D=10-30 \mathrm{~km}$ bodies present in the prograde population at $t=500 \mathrm{Myr}$. Low impact energies also mean cratering events are more common than before. As seen in the $t=500 \mathrm{Myr}, 1000 \mathrm{Myr}$, and $1500 \mathrm{Myr}$ time steps, the fragment tail of this ejecta shows up for $D<1-7 \mathrm{~km}$ and will actually wither away on timescales of tens of millions of years until a stochastic disruption or cratering event on one of the larger remaining satellites freshens it up with new ejecta. The pattern then begins again. By animating the timesteps, we find the $D<7 \mathrm{~km}$ SFDs for both populations wave up and down again and again over billions of years, much like a ringmaster cracking a whip.

Our best score for this case comes at $t=3651$ Myr. The $S$ values for the prograde and retrograde SFDs are 0.15 and 0.07 , quite good, but with some limitations that we will discuss below. Here, ejecta evolving from a cratering event on the largest remaining retrograde satellite reproduces the observed steep retrograde population near $D \approx 7 \mathrm{~km}$. This excellent match does not last more than a few tens of Myr, but comparable fits are found at several later times in the simulation via the "cracking whip" process described in the previous section.

The main deficiencies in this trial case are twofold. First, our model was unable to reproduce the substantial difference in diameter between Phoebe and the second largest retrograde irregular satellite (Figure 10). Second, our model prograde satellites for $D>30 \mathrm{~km}$ are modestly larger than the observed ones. Similar problems show up in other $S<0.3$ trial cases.

The culprit is likely to be our initial conditions. Using our selected capture efficiencies, source populations A and B (Figure 3) nearly always produce a continuum of retrograde objects, which makes it difficult to produce significant differences in size between Phoebe and the second-largest retrograde object. Similarly, if all captured objects follow the same $Q_{D}^{*}$ disruption function, it is difficult to eliminate large numbers of middle-sized objects without affecting Phoebe as well.

The solution to the Phoebe problem is unclear, but we hypothesize that it involves factors that we have yet to consider in our modeling work. For example, Phoebe, classified as a C-type object with water ice and $\mathrm{CO}_{2}$ on its surface (Johnson \& Lunine 2005) as well as a bulk density of $1.634 \mathrm{~g} \mathrm{~cm}^{-3}$ (Jacobson et al. 2006), may be harder to disrupt than typical D- and P-type irregular satellites. In this scenario, the smaller objects would disrupt/erode while Phoebe would be left more or less intact. For a second possibility, it could be that the capture efficiency at Saturn was lower than at the other gas giants, and Phoebe's capture was a fluke at the $\sim 10 \%$ level. This would allow fewer middle-sized objects to be captured. A third possibility is that Phoebe was captured onto its current orbit in a different manner than the other irregular satellites (e.g., perhaps the binary capture mechanism that allowed Triton to be captured at Neptune; Agnor \& Hamilton 2006; Vokrouhlický et al. 2008). The problem is finding a mechanism that works during or after the Nice model and allows Phoebe to reach its current orbit (Vokrouhlický et al. 2008). Note that these scenarios do not have to be mutually exclusive; perhaps several of them played a role at some level.

The top five irregular satellite runs for Saturn are somewhat more varied than those for Jupiter (Table 3). Good fits between model and data occurred $25 \%-40 \%$ of the time over the last $500 \mathrm{Myr}$ of the simulations. We believe this fractional value, while lower than Jupiter's by a factor of 1.4, is still highly encouraging, particularly when one considers the Phoebe issue above as well as how difficult it is to fit the always-evolving small end of the SFDs for long intervals. The combined results shown in Figures 8 and 9 indicate that Saturn's runs follow the same overall trends as Jupiter's runs. Moderately favored parameters are $f_{Q}$ values of $3-8, N(D>250 \mathrm{~km})$ values of $0.1-1.0$, variants of population A ( $q$ values of -5.5$), f_{\text {split }}$ values of 0.5 , and $M_{\text {disk }}$ values of $40 M_{\oplus}$. These results are consistent with the trends seen in the top five runs, where normalization values of $N(D>250 \mathrm{~km})=0.1$, population A input SFDs $(q=-5.5)$, and larger disks $\left(M_{\text {disk }}=40 M_{\oplus}\right)$ are favored. 


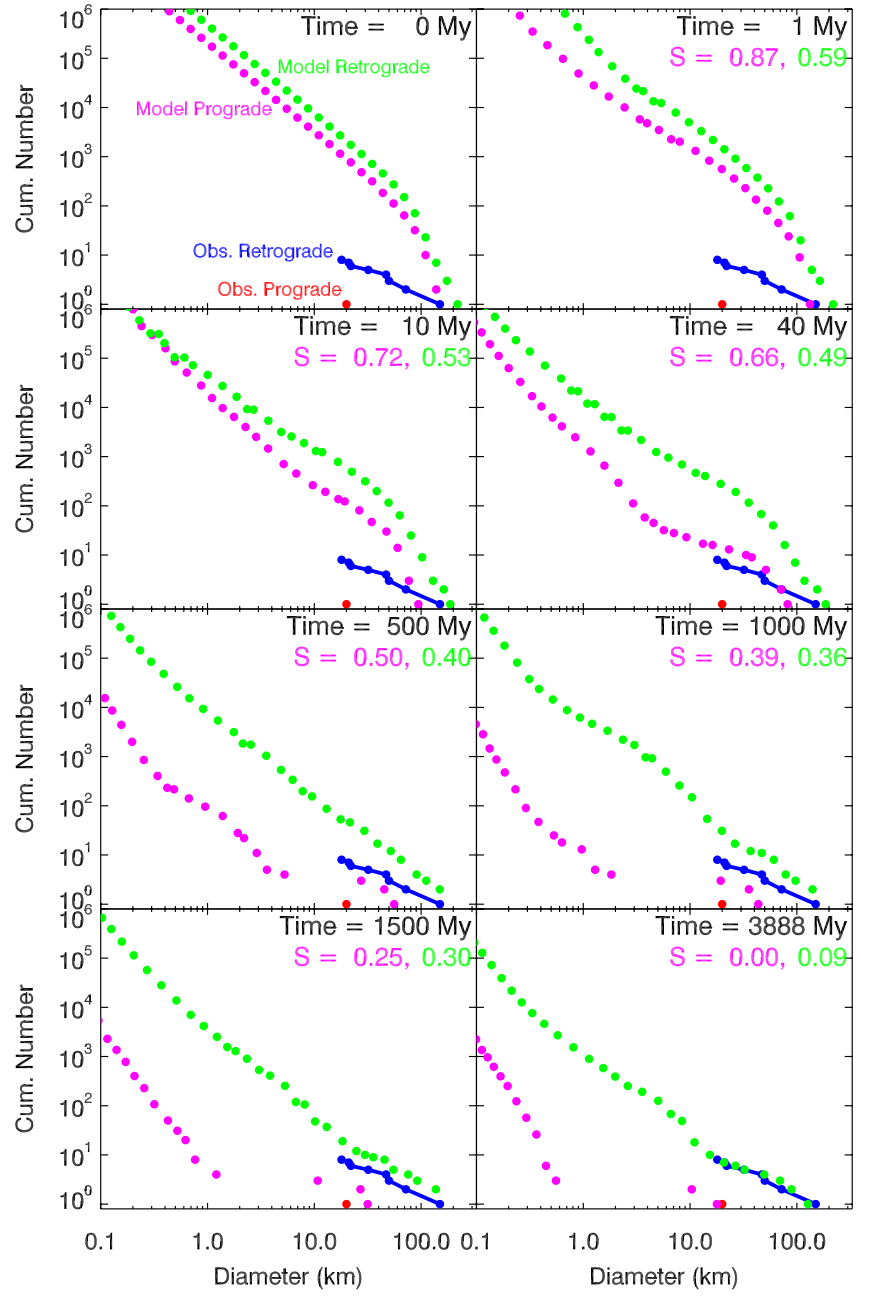

Figure 11. Collisional evolution of Uranus's irregular satellites. The color code and labels are the same as in Figure 7. The input parameters used were $f_{Q}=8$, $f_{\text {split }}=0.3, N(D>250 \mathrm{~km})=1.0, q=-2.0$, and $M_{\text {disk }}=20 M_{\oplus}$. Note that the $f_{\text {split }}=0.3$ value is lower than those used for the Jupiter and Saturn trial cases. We see that the initial differences between the prograde and retrograde SFDs become more and more pronounced as time goes on, with the larger retrograde population obliterating the prograde one. After $500 \mathrm{Myr}$, only a few prograde objects with diameter $D>20 \mathrm{~km}$ are left. At that point, cratering events on these objects over billions of years reduce their mass to such a degree that the single largest model prograde object eventually matches the observed one. The match for the retrograde objects is also remarkable, with most of the collisional evolution over the last few billion years occurring via retrograderetrograde impacts.

\subsection{Uranus's Irregular Satellites}

The irregular satellites of Uranus have SFDs that are somewhat different than those examined thus far (Figure 1). The known prograde population is comprised of a single $D=20 \mathrm{~km}$ object, while the retrograde SFD is a fairly smooth continuum made up $10 D>10 \mathrm{~km}$ objects and $1 \mathrm{D}>100 \mathrm{~km}$ object. This order of magnitude mismatch implies something interesting went on in this system. A partial cause may be the low $V_{\text {imp }}$ values for satellite collisions, which range between $1 \mathrm{~km} \mathrm{~s}^{-1}$ and $2 \mathrm{~km} \mathrm{~s}^{-1}$ (Table 2). This is unlikely to be the whole story, though, because Saturn's values are only modestly higher.

Figure 11 shows eight snapshots from a trial case with a very good fit to Uranus's irregular satellites. The input parameters used were $f_{Q}=8, f_{\text {split }}=0.3, N(D>250 \mathrm{~km})=1.0$, $q=-2.0$, and $M_{\text {disk }}=20 M_{\oplus}$. The $f_{\text {split }}=0.3$ value used here is lower than those used for the Jupiter/Saturn cases but justifiable based on the dynamical capture runs of Nesvorný et al. (2007). We were driven to this value by multiple test runs using $f_{\text {split }}$ values of 0.4 or 0.5 ; neither choice produced more than a marginal number of successful fits among our many trial cases.

We found that in this trial case, the "head start" given to the retrograde SFD over the prograde one allowed it to immediately dominate the collisional evolution of the system. This leads to what we refer to as a runaway collisional process. The prograde SFD quickly becomes decimated, so much so that its fate is controlled by the evolution of the retrograde SFD. This leads to the endgame situation seen at $t=500 \mathrm{Myr}$, where only a handful of large prograde objects are left standing.

After $500 \mathrm{Myr}$, the remaining retrograde SFD (and external Kuiper Belt/scattered disk SFDs) are depleted enough that collisional erosion among the prograde objects is dominated by cratering rather than catastrophic disruption events. Bit by bit, these relatively small impacts, over billions of years, grind down the surviving prograde objects. Eventually, at $t=3888 \mathrm{Myr}$, we end up with an excellent fit between the model and observed SFDs (i.e., $S=0.0005$ for prograde objects and $S=0.09$ for retrograde objects). The high quality of our fit, and the fact that the prograde population only includes one $D \approx 20 \mathrm{~km}$ object, allows us to make the following predictions: (1) by the end of the capture phase, the retrograde population was substantially larger than the prograde one, and (2) the initial difference between the SFDs was greatly exacerbated over billions of years by a runaway collisional process that produced the near-elimination of the prograde population.

The odds of getting a runaway collisional outcome are reasonable but not overwhelming. From our top five irregular satellite runs for Uranus (Table 3), we find success rates of 10\%$30 \%$ when we checked the last $500 \mathrm{Myr}$ of our simulations. These results also favor larger initial populations (i.e., $N(D>$ $250 \mathrm{~km}$ ) values of 1.0), population A-type SFDs (i.e., $q$ values of -5.5 ), and satellites that are easy to disrupt (i.e., $f_{Q}$ values of 8). Using the combined results shown in Figures 8 and 9, we obtain similar results. There are relatively strong preferences for $f_{Q}$ values near 8 and $N(D>250 \mathrm{~km})$ values near 1.0 . The preferences are more moderate for $M_{\text {disk }}$ values near $20 M_{\oplus}$ and $q$ values near -5.5 .

\section{CONCLUSIONS}

Overall, our results indicate that the irregular satellite populations immediately after capture were likely to have been significantly larger than what we observe today. These populations then experienced rapid collisional evolution and almost literally self-destructed. After several hundreds of Myr, the SFDs in different irregular satellite systems evolved from our assumed Jupiter Trojan-like SFDs to quasi-steady-state SFDs with extremely shallow power-law slopes for $D>10 \mathrm{~km}$ objects (e.g., Figure 2). The severe mass depletion in these populations prevents the larger survivors from experiencing a high rate of catastrophic disruption events. This means that the remnant populations change very slowly over $\sim 3.5 \mathrm{Gyr}$ of evolution. We believe this explains why the combined prograde and retrograde irregular satellite SFDs are so similar to one another. These results also show that the starting time of the simulations does not affect the results in a meaningful way; if the Nice model had started at $3.5 \mathrm{Ga}, 3.9 \mathrm{Ga}$, or $4.5 \mathrm{Ga}$, the results would be largely the same.

Our results also indicate that the Nice model framework has passed another critical test. Even though the Trojans and irregular satellites have remarkably different SFDs, they could 
have and probably did come from the same source population (i.e., the primordial trans-planetary disk, the source of many other small body populations; Levison et al. 2009; Morbidelli et al. 2009b). This strengthens the likelihood that the Nice model is producing reasonable results and raises the bar for alternative models of solar system evolution.

Additional take-away results from our model runs are briefly summarized below.

1. We assumed that the irregular satellite populations were similar in many ways to the Jupiter Trojan SFD seen today (see Levison et al. 2009). We cannot claim, however, that this is the only possible starting SFD. Given how our model irregular satellite populations evolve over time, we suspect other input SFD could produce similar results (i.e., one could imagine capturing a SFD with a shape similar to one of the intermediate steps seen in Figures 7, 10, and 11). Some of the constraints on alternative initial SFDs would be: (1) they need to be large enough to produce the largest observed irregular satellites, and (2) they cannot contain too many large objects or we would see them today; collisions alone do not appear capable of getting rid of the evidence.

2. Irregular satellites captured from the primordial transplanetary disk disrupt at much lower impact energies than stony main Belt asteroids. Our model results for the collisional evolution of irregular satellites at Jupiter, Saturn, and Uranus show preferences for $f_{Q}>3$, with the most favored values being $f_{Q}=3,5$ (Table 3; Figure 8). Interestingly, $f_{Q}=3,5$ were also favored by Levison et al. (2009), who modeled the collisional and dynamical evolution of captured primordial trans-planetary disk objects within the outer main Belt, Hilda, and Trojans populations and collisional evolution within the primordial trans-planetary disk itself. The self-consistency of these results implies that we have accurately identified some of the key physical properties of comet-like objects.

3. Our model results show a moderate preference for input SFDs that look like population A over population B. Trial cases suggest this is because Phoebe-sized and larger objects are hard to eliminate by collisional evolution (i.e., if they were placed there long ago, they or their fragments would be observable there today). Therefore, if the source SFD from the primordial trans-planetary disk had a "foot," it presumably would need to exist at sizes much larger than $D>250 \mathrm{~km}$; this would allow very large objects to avoid capture in the irregular satellite and Trojan asteroid systems.

4. Our model results predict that the size of the captured satellite population at Uranus is likely to be a factor of 3-10 higher than those at Jupiter or Saturn. Both the top five results in Table 3 and the combined parameter results in Figure 8 show that irregular satellite populations at Uranus favor normalization $N(D>250 \mathrm{~km})$ values near 1.0, while those at Jupiter and Saturn favor near 0.1-0.3. This is very consistent with the predictions of Nesvorný et al. (2007), who found that Uranus and Neptune have more planetary encounters than Jupiter and Saturn.

5. Our collision evolution results do not appear to be strongly dependent on impactors that come from the primordial trans-planetary disk. Tests using disk masses (at the time of the LHB) of $M_{\text {disk }}=20$ and $40 M_{\oplus}$ produce no preference in our model results (Figure 9). This makes sense if one considers that most of the collisional evolution comes from the irregular satellites themselves; disk impactors mainly act as a perturbation on that signal. Disk impactors can be important at later times, though, when large stochastic breakup events are capable of significantly affecting the SFD of a collisionally depleted irregular satellite population.

6. Model runs indicate the irregular satellite populations at Jupiter and Saturn likely started with comparable numbers of prograde and retrograde satellites. At Uranus, however, we need a 70-30 split or higher in favor of the retrograde population to produce the observed mismatch of retrograde/prograde satellites.

7. Our model has the greatest difficulty fitting the largest bodies in the Saturnian system and the large gap in size between Phoebe and the second-largest retrograde object. We hypothesized several different ways to explain this: (1) C-type Phoebe may be more difficult to disrupt than standard D/P-type irregular satellites (e.g., it is more stony than typical irregular satellites); (2) Saturn's satellite capture efficiency may have been lower than predicted here, with Phoebe's capture a fluke; and (3) Phoebe's dynamical history may be distinct from other irregular satellites.

\section{IMPLICATIONS}

\subsection{Did the Irregular Satellites Cover the Outermost Regular Satellites with Dark Dust?}

An interesting and potentially exciting implication of our model results is that the same collisional cascades that demolish C-, D- and P-type irregular satellites should also create enormous amounts of dark dust. For example, using the starting SFDs shown in Figures 7, 10, and 11, we predict that $~ 99 \%$ of the mass of the irregular satellite populations at Jupiter, Saturn, and Uranus were lost to comminution over $4 \mathrm{Gyr}$. Thus, in each case, we have $\sim 0.001$ lunar masses or more of dust to deal with. What happened to this material?

Small particles in the solar system are strongly affected by solar radiation pressure forces and Poynting-Robertson (P-R) drag (for a review of these processes, see Burns et al. 1979). The importance of these mechanisms depends on the size, shape, and composition of the particles. Burns et al. (1979) show that micron-sized dust particles on planetocentric orbits have their eccentricities rapidly pumped up by radiation pressure forces, while P-R drag sends objects up to hundreds of microns in size inward toward the planet. If started from present-day irregular satellite orbits, these particles will eventually achieve crossing orbits with the outermost regular satellites, where they have the potential to strike them.

The collision probabilities between irregular satellite dust and the regular satellites have only been calculated for a limited number of examples, probably because few irregular satellites were known until recently. The most prominent cases in the literature involve dust particles from Phoebe evolving inward to strike Iapetus $(D=1470 \mathrm{~km})$, the outermost regular moon of Saturn with a dark leading side and a bright trailing side. Burns et al. (1996) estimated that $D=20 \mu \mathrm{m}$ sized particles from Phoebe have a $70 \%$ chance of striking Iapetus. Those that get past Iapetus have a $60 \%$ chance of striking Hyperion $(D=$ $270 \mathrm{~km})$. The rest are lost to Titan $(D=5150 \mathrm{~km})$, which protects the inner moons from significant dust contamination. Burns et al. report that smaller and faster-moving particles have lower impact probabilities with Iapetus and Hyperion, but few still get past Titan. The equations provided by Burns et al. (1979) indicate comparable impact probabilities should exist between dust particles from irregular satellites and the outermost regular 
satellites of Jupiter and Uranus. Part of the reason has to do with target size; at Jupiter, Callisto and Ganymede $(D=4820$ and $5260 \mathrm{~km}$, respectively) are similar in size to Titan and are much larger targets than Iapetus, while at Uranus, Oberon and Titania $(D=1520$ and $1580 \mathrm{~km}$, respectively) are only slightly larger than Iapetus.

The inescapable conclusion from all of this is that the outermost regular satellites of the gas giants should have been blanketed by large amounts of dust produced by irregular satellite comminution. Most of this dark material would have landed within the first few hundreds of Myr after the capture of the irregular satellites. Thus, if the timing of the Nice model is linked with the ages of late-forming lunar basins like Serentatis and Imbrium, which have ages of $\sim 3.9$ Ga (Stöffler \& Ryder 2001), most of this dark dust landed on the satellites $\sim 3.5$ 3.9 Gyr ago.

Many authors have either suggested or explored variants of this intriguing scenario over the last several decades. While this is by no means a complete list, the interested readers are encouraged to investigate the following papers and references therein (e.g., dynamical links between Phoebe dust and the leading face of Iapetus; Soter 1974; Burns et al. 1996; dark nonicy components with spectroscopic properties similar to C-, D-, and P-type asteroids have been identified on many outermost regular satellites; Cruikshank et al. 1983; Buratti \& Mosher 1991, 1995; Buratti et al. 2002; meteoroid contamination from the irregular satellites may be the source of the albedo differences on Ganymede and Callisto; Pollack et al. 1978; Johnson et al. 1983; Bell et al. 1985; see also McKinnon \& Parmentier 1986). Presenting all of this material is beyond the scope of this paper, so below we focus on several interesting regular satellites.

\subsubsection{Callisto}

According to the review chapter by Moore et al. (2004; see also McKinnon \& Parmentier 1986), Callisto's reflectance spectrum appears to be a combination of water-ice and a dark non-icy material that may be similar to carbonaceous chondrites. Callisto's surface is either bright (albedos near 0.8) or dark (albedo near 0.2), with little in between. Away from opposition, the trailing side is $12 \%$ brighter than the leading side, which is opposite the pattern on the other Galilean satellites. Bright material is found on the crests of high standing topography, while dark material is almost always found in low-lying areas. Sizable young impact craters often have bright centers and rays. These craters appear to have punched through a few kilometers of regolith contaminated by dark material to reach a clean ice zone.

As first argued by Pollack et al. (1978; see also Burns et al. 1979), these conditions appear consistent with the idea that Callisto currently has a veneer of dark material that was largely put in place billions of years ago. A back of the envelope calculation indicates that 0.001 lunar masses of irregular satellite dust transferred with high efficiency to Callisto could produce a surface layer $300 \mathrm{~m}$ thick. This quantity of material, if mixed into the upper few kilometers of Callisto's crust by impacts, may explain Callisto's observational constraints. Any dark dust that slips by Callisto and reaches Ganymede may explain its ancient dark terrains as well (J. Moore 2009, private communication; see also Pappalardo et al. 2004).

The observation that Callisto's leading side is slightly darker than its trailing surface may also tell us something interesting about Callisto's evolution. If a 50-50 mix of prograde and retrograde dust on modestly eccentric orbits were to cross Callisto's path, the majority of the material should strike on Callisto's leading hemisphere (i.e., most retrograde material can only strike the leading face, while the prograde material can hit both the leading and trailing sides). Note that this assumes that Callisto has always been in synchronous rotation, which is true today but may not have been true for all of Callisto's history. If it had, Callisto would probably be far darker on its leading side than its trailing side. Thus, to explain Callisto's limited color asymmetry, Callisto likely had to escape synchronous lock several times near the peak of the dust flux.

How could this happen? New modeling work on our Moon suggests that large basin-forming events may have allowed it to break synchronous lock with the Earth long enough for the leading/trailing faces to flip prior to being recaptured again (Wieczorek \& LeFeuvre 2009). It is possible that the same mechanism is applicable to Callisto, though we caution that this strongly depends on our limited knowledge of its principal moments of inertia (Lissauer 1985) as well as how Callisto internally evolved in reaction to being struck again and again by impactors during the late heavy bombardment (Barr \& Canup 2010).

Thus, by combining our dust flux model with a dynamical model of dust evolution, we may be able to use leading/trailing albedo differences across Callisto like a clock to determine the approximate age of its youngest, largest basin.

\subsubsection{Iapetus}

Many authors have written about the dramatic brightness asymmetries found between Iapteus's leading face, which is largely dark, and its trailing side, which is largely bright. Focusing on extrinsic sources, Soter (1974) and others (see references above) have suggested variants of the following model: retrograde dust from Phoebe and possibly other irregular satellites are the source of this hemispherical black and white difference, with dark retrograde dust preferentially swept up on the leading side by an Iapetus in synchronous rotation. Our contribution to this scenario would be that dark dust from many different ground-up non-Phoebe irregular satellites would circumvent the problem that Iapetus's darkest terrain does not look like C-type Phoebe itself (e.g., Burns et al. 1996).

This solution, while dynamically elegant, has certain issues. One problem is that the poles are largely white when they should be covered with dark material (e.g., Burns et al. 1996). A second problem is that Iapetus should not be that different than Callisto, at least in terms of exposure to large quantities of prograde and retrograde dust as well as basin-forming impactors capable of breaking its synchronous lock with Saturn (though Iapetus may have taken a billion years or so to achieve synchronous lock; Hamilton 1997). Moreover, our model predicts the dust layer should be substantial; if 0.001 lunar masses of irregular satellite dust was transferred to Iapetus with 10\%-100\% efficiency, it would produce a surface layer $0.3-3 \mathrm{~km}$ thick. Thus, like Callisto, our expectation is that Iapetus should be blanketed on all sides with dark material, with perhaps a small preference for dark dust on its current leading side. This is not observed.

A possible solution to these problems may come from Spencer et al. (2005; see also Denk \& Spencer 2008), who argued that Iapetus's long rotation period (79 days) allows water to bake out of the surface on the dark side and migrate to colder climes on the trailing side and at the poles (see also Hamilton 1997 for a variant of this idea). This runaway process, over a timescale on the order $100 \mathrm{Myr}$, would potentially create the dichotomy seen 
today. The trigger for this mechanism would be the dark non-icy material raining down from the irregular satellites. In a similar fashion, the dark material found on Hyperion would originate from irregular satellite dust that managed to avoid Iapetus (e.g., Burns et al. 1996).

\subsubsection{Titan}

Titan is Saturn's largest satellite $(D=5150 \mathrm{~km})$. Only tiny Hyperion $(D=270 \mathrm{~km})$ and Iapetus $(D=1470 \mathrm{~km})$ stand between it and the irregular satellite population. This means that, as suggested by Burns et al. (1996), it should be a major sink for dark dust dynamically evolving inward toward Saturn. The question is whether obvious signs for this material exist on Titan's surface.

The bulk composition of Titan, like most outer planet satellites, is thought to be largely composed of water ice and rock. Where Titan differs from its brethren is in its thick atmosphere of nitrogen and hydrocarbons, which allows it to have geologic land forms that are unique among the outer planet satellites (e.g., hydrocarbon lakes, channels presumably formed by rain and flowing liquids). In particular, some of the most unexpected and mysterious features on Titan are its equatorial longitudinal dune fields that cover $20 \%$ of its surface (Lorenz et al. 2006). According to press reports, the dunes appear to be similar in appearance to "mountainous drifts of coffee grounds;" they are radar and optically dark with spectral shapes consistent with carbon-rich materials (Barnes et al. 2008).

Most groups to date have concentrated on endogenous models for dune particle formation. This is tricky, though, because Titan's dune particles need to have several specific attributes. First of all, they have to contain less water ice than the rest of Titan's surface (Barnes et al. 2008). Second, the presence of longitudinal dunes implies Titan's dune particles act like common sand in Titan's winds. On Earth, longitudinal dunes are created by the process of saltation, where coarse, non-sticky, erosion-resistant particles "hop", or are temporarily suspended in winds before landing in a nearby location. Thus, to move in Titan's prevailing equatorial winds, Titan's dune particles need to have these characteristics as well as diameters near $200 \mu \mathrm{m}$ (Lorenz et al. 2006; Barnes et al. 2008). Finally, Titan needs to have some way of producing a lot of dune particles; current estimates suggest the total volume of the dune fields is $>2 \times 10^{5} \mathrm{~km}^{3}$ (Lorenz et al. 2008).

Some argue that Titan's dune particles formed via a twostep process: first, precipitation created tiny organic grains that then grew larger on the ground through sintering processes over $10^{4}-10^{8}$ yr (Brown et al. 2006; Barnes et al. 2008). It is unknown, however, whether such a process can work or whether the resultant particles could reproduce the constraints described above. Others claim that the material could come from crater ejecta or eroded material from Titan's observed river channels. The problem, as pointed out by Lorenz et al. (2008), is that neither mechanism is capable of reproducing the observed volume of the dune fields.

We postulate that a simpler and perhaps more straightforward solution is an exogenous one, namely that that Titan's dune particles were created by the collisional demolition of Saturn's irregular satellites. This model is not only consistent with the constraints described above but also with our expectations based on observations of Callisto, Ganymede, Iapetus, Hyperion, and the Uranian satellites (see below). Consider the following: (1) The total volume of the dune fields, $>2 \times 10^{5} \mathrm{~km}^{3}$, is only $\sim 1 \%$ of predicted starting volume of the irregular satellites $(\sim 0.001$ lunar masses). Thus, the irregular satellites can readily produce enough material to create the dunes, even if some material is lost in transit or by early Titan geology. (2) The dark nonicy component seen on the outermost regular satellites appears similar to primitive carbonaceous chondrite-like meteorites (e.g., Orgueil; Tagish Lake). If one were to grind up such meteorites, the resultant crop of sand particles would largely reproduce what we know of Titan's dune particles, particularly if Titan's atmosphere coated them with a rind of organics (e.g., Barnes et al. 2008).

Considerable work remains to prove this hypothesis. If true, however, this scenario has critical implications for our understanding of Titan's history. For example, Figure 10 indicates that most of Saturn's irregular satellites were ground down by collisions within a Gyr or so of capture. This leaves little margin to replenish the observed dune volume of $>2 \times 10^{5} \mathrm{~km}^{3}$ over the last several Gyr if significant quantities of dune particles were eliminated by geologic processes. Thus, the presence of widespread dunes could strengthen claims that that Titan has been, for the most part, geologically inactive for the last several Gyr (see Moore \& Pappalardo 2008).

\subsubsection{Uranian Satellites}

None of the large regular Uranian satellite stand out in the same way as Callisto or Iapetus. From the inside out, they are Miranda, Ariel, Umbriel, Titania, and Oberon $(D=470 \mathrm{~km}$, $1160 \mathrm{~km}, 1170 \mathrm{~km}, 1580 \mathrm{~km}$, and $1520 \mathrm{~km}$, respectively). These bodies are dark, grayish worlds that appear to be mixtures of water-ice and a dark, non-icy spectrally gray to reddish material (Veverka et al. 1991; Buratti \& Mosher 1991). Their surface albedos, except for Umbriel, vary by at least a factor of 2, with the highest values corresponding with impact craters, ejecta deposits, and other geologic features (Veverka et al. 1991). Buratti \& Mosher (1991) report that the outer four bodies have leading/trailing asymmetries in both their color and albedo. In fact, as one moves out in the system, the trailing sides of each satellite becomes progressively redder. Only Miranda, the innermost regular satellite, appears to be free of the contaminant that has affected the outer regular satellites. From this, Buratti \& Mosher (1991) argue that the data are most consistent with a scenario where the satellites accrete low albedo, gray to reddish dust particles from a population of irregular satellites.

We suspect the differences between the satellites probably come from attrition; as dust from the irregular satellites evolves inward, most of it is scooped up by Oberon, Titania, etc. In the end, very little gets to Miranda. In addition, the observed bright features and differences in dust contamination probably come from impact craters or geologic events that have managed to dredge up clean ice located below the contaminated regolith.

\subsubsection{Summary and Discussion}

Observations of the outermost regular satellites of Jupiter, Saturn, and Uranus appear consistent with significant surface contamination by a dark, non-icy material with physical properties similar to C-, D-, and P-types asteroids. We strongly suspect that the source of this material was dust produced by collisional evolution among the irregular satellites whose original mass was $\sim 0.001$ lunar masses. Most of the dust was created within the first few hundreds of Myr after capture. Once created, it migrated inward toward the central planet and struck the outermost satellites. Other than Iapetus, no satellites show strong leading/ trailing side albedo asymmetries. This could be from two reasons; the initial irregular satellite systems had large prograde and 
retrograde populations, allowing impacts on the satellites from many directions, and impacts or other mechanisms (e.g., slow tidal evolution) may have allowed the satellites to stay free from or break synchronous rotation when the dust infall was near its peak. The more limited leading/trailing asymmetries seen today may be a byproduct of a more limited dust flux that struck synchronously rotating satellites over the last several Gyr. The significant color differences seen on Iapetus may be a byproduct of volatile movement from the warmer to colder climes and the development of frost.

We close this section by probing whether the dark dust on the regular satellites could come from heliocentric sources (i.e., collisions within the primordial trans-planetary disk and/or among objects in the Kuiper Belt/scattered disk populations). We believe the telltale parameter here is the gravitational focusing factor of the gas giants, which strongly increases the flux of heliocentric material passing near the inner moons relative to the outer moons.

Using the (simplistic) equations found in the appendix of Richardson et al. (1998), we computed the ratio of heliocentric dust striking a square kilometer of Tethys and Rhea (semimajor axis of $a=294,619 \mathrm{~km}$ and $527,719 \mathrm{~km}$, respectively) to that of Iapetus ( $a=3,560,820 \mathrm{~km})$. We chose Tethys, Rhea, and Iapetus because they are heavily cratered (e.g., Kirchoff \& Schenk 2009) and roughly similar in size. This implies they are likely to have comparable surface ages. Unlike Iapetus, however, Tethys and Rhea show few signs of a significant dark nonicy carbonaceous chondrite-like component (Verbiscer et al. 2007). Our test heliocentric dust particles were assumed to enter Saturn's system on hyperbolic orbits with velocities of 1-5 $\mathrm{km} \mathrm{s}^{-1}$ (e.g., Levison et al. 2000).

We found that heliocentric dust particles are at least 4 10 times more likely to strike Tethys or Rhea than Iapetus. These values are inconsistent with observations. These dust flux ratios are also lower limits because the equations in Richardson et al. (1998) ignore the orbital velocities of the satellites; inner moons like Tethys and Rhea revolve fast enough around Saturn that their intrinsic collision probabilities with heliocentric objects are one to two orders of magnitude higher than Iapetus (e.g., Bottke et al. 1994). We conclude from this that dark dust from the irregular satellites is more likely to be a major contributor to the surfaces of regular satellites than heliocentric dust and is more consistent with observations.

\subsection{Regular Satellite Craters Produced by the Irregular Satellites}

Another intriguing implication of this work concerns the crater records of the outermost regular satellites. Most groups have tried to interpret and/or model these crater populations using heliocentric impactors (e.g., refugees from the primordial trans-planetary disk, ecliptic comet, and nearly isotropic comet populations) or planetocentric ones (e.g., planetesimals leftover from satellite accretion; fragments from the catastrophic disruption of a small moon; ejecta from cratering events on the regular satellites; refugees from the asteroid Belt that somehow enter into planetocentric orbits prior to satellite impact; e.g., Strom 1987; Chapman \& McKinnon 1986; McKinnon et al. 1991; Levison et al. 2000; Zahnle et al. 1998, 2001, 2003, 2008; Schmedemann et al. 2009; Charnoz et al. 2009). No group has yet considered, however, whether irregular satellites constitute an important source of impactors for the regular satellites.

Immediately after capture, the irregular satellites, whose population should rival the size of the present-day Trojan asteroids (Figure 2), comprise a nearly isotropic distribution of impactors spanning much of the space within each giant planet's Hill sphere (Nesvorný et al. 2007). Several sub-populations within this group are capable of striking the regular satellites. For example, objects embedded in the Kozai resonance will move into the regular satellite region on $10^{4} \mathrm{yr}$ timescales (Carruba et al. 2002; Nesvorný et al. 2003). Another fraction, captured outside the regular satellite-crossing zone but inside the stable irregular satellite zone, will move more slowly onto collision trajectories with the regular satellites (e.g., Carruba et al. 2002; Nesvorný et al. 2003; Haghighipour \& Jewitt 2008). Finally, impacts on irregular satellites from irregular or heliocentric impactors may inject new fragments onto unstable orbits where they can then go on to strike the regular satellites. The latter source may create some craters on the regular satellites today.

Irregular satellites capable of striking the regular satellites should have SFDs that change significantly over time via collisional and dynamical evolution (e.g., Figures 7, 10, and 11). This means that if the irregular satellites are important sources of craters, the chronology of events on the outer planet satellites can only be accurately interpreted using a coupled collisional and dynamical evolution model. While a full test of this scenario is beyond the scope of this paper, we decided that, as a proof of concept test, it would be interesting to see whether the shapes of the crater SFDs found on some outer planet satellites are similar to "snapshots in time" of the SFDs found in our best-fit irregular satellite trial cases (Figures 7, 10, and 11).

Our results for Callisto (at Jupiter), Iapetus (at Saturn), and Titania (at Uranus) are shown in Figure 12. The crater counts and error bars were taken from the literature: Callisto (Strom et al. 1981; McKinnon et al. 1991), Iapetus (Jaumann \& Neukum 2009), and Titania (Strom 1987). The crater data were converted into cumulative projectile SFDs using the scaling laws described by Zahnle et al. (2001). The impact velocities between regular and irregular satellites were computed using the code of Bottke et al. (1994), with our accepted values essentially the same as those reported in Zahnle et al. (2001) for heliocentric impactors. Note that because the absolute scaling is unknown, the transformed crater SFDs were slid up and down and fit "by eye" to the dominant irregular satellite SFD (usually retrograde) every 1 Myr over 4 Gyr of evolution. The best overall fit between model and data for each case is shown in Figure 12.

Overall, the matches are surprisingly good, especially when one considers our unknowns and that we are attempting to compare crater densities, which are integrated over the age of a surface, to snapshots in time from our Boulder trial cases. Thus, if the fits are not flukes, they may be telling us about the evolution of an irregular satellite population captured on unstable orbits (Callisto, Iapetus) or how stochastic breakup events at particular epochs dominate the crater records of some satellites (Titania). They also present us with a tantalizing possibility that the shape of a crater SFD alone may be diagnostic enough to estimate surface ages. On this basis, we analyze our model results below.

For Callisto, the best fit to the crater SFD comes at $t=$ $25 \mathrm{Myr}$, where $t$ is defined as the time after the initial capture of the irregular satellites (Figure 12). Interestingly, this age is broadly consistent with Levison et al. (2002), who claimed that the clearing of the primordial trans-planetary disk should have produced enough basin-forming impacts to melt Callisto's surface to considerable depth (see also Barr \& Canup 2010). If true, our predicted surface age may represent the time Callisto's surface had stabilized enough to support impact structures again. 


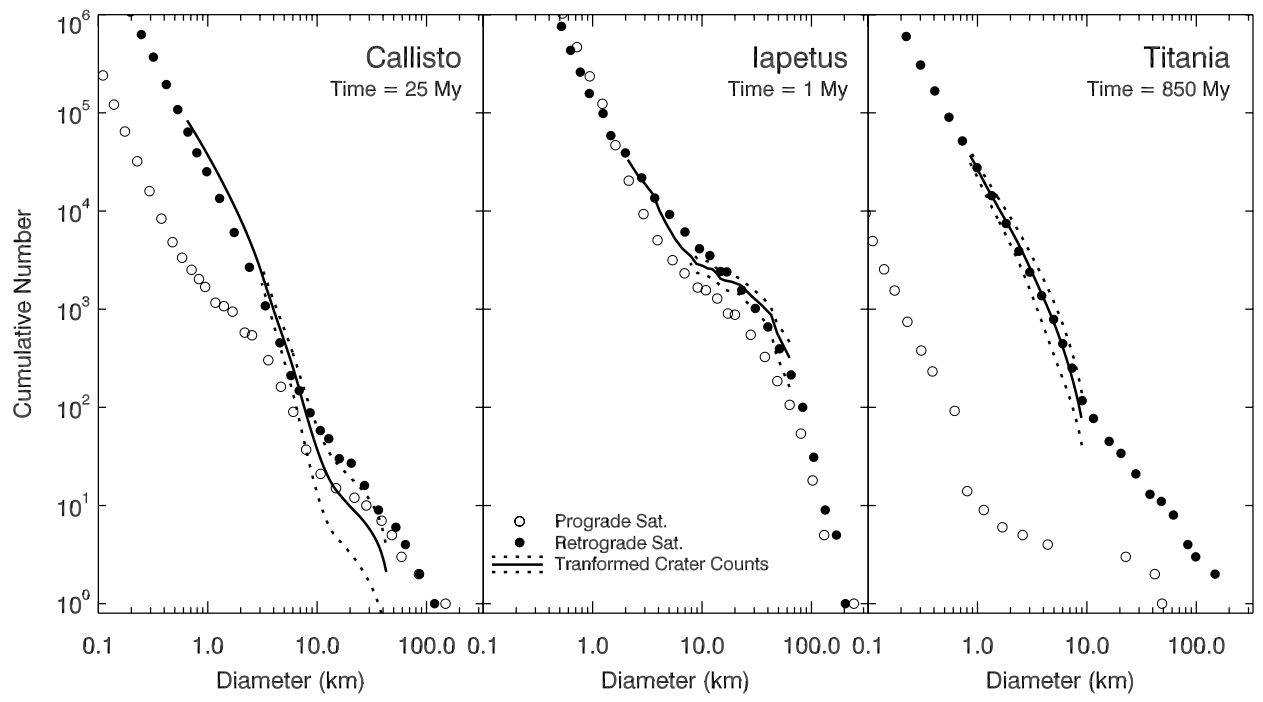

Figure 12. Comparison between the crater SFDs on Callisto, Iapetus, and Titania and snapshots of the irregular satellite SFDs for Jupiter, Saturn, and Uranus, respectively. The $x$-axis is the object diameter (in kilometers) and the $y$-axis is the cumulative number. The crater counts and error bars, represented by the solid and dashed lines, were transformed into irregular satellite sizes using scaling relationships from Zahnle et al. (2001). The filled and open circles are model retrograde and prograde irregular satellites, respectively, taken from the trial cases described in Figures 7, 10, and 11. The best fits between the crater data and the dominant irregular satellite populations were estimated "by eye." The time of irregular satellite capture corresponds to $t=0$ Myr. In absolute time, this may approximately correspond to $3.9 \mathrm{Ga}$, the formation time of the lunar basins Imbrium and Serenitatis with solid age constraints (Stöffler \& Ryder 2001).

Iapetus may have the oldest non-saturated surface among the outer planet satellites. Using a projectile SFD for the primordial trans-planetary disk based on Bernstein et al. (2004), Charnoz et al. (2009) showed that heliocentric impactors during the Nice model may be a primary source of basins and $D=10 \mathrm{~km}$ craters on Iapetus's surface. The crater SFD for $D<10 \mathrm{~km}$ craters, however, is weakly " $\mathrm{S}$ "-shaped (Figure 12); our results indicate that such shapes are diagnostic of irregular satellite populations that have experienced a short interval $(t \sim 1 \mathrm{Myr})$ of intense collision evolution. At this time, we cannot say whether Iapetus's largest basins were produced by heliocentric or planetocentric impactors, but we find it probable that many smaller craters come from irregular satellites.

Titania is the largest moon of Uranus $(D=1578 \mathrm{~km})$ but is comparable in size to Oberon $(D=1523 \mathrm{~km})$ on the outside and is slightly larger than Umbriel $(D=1169 \mathrm{~km})$ on the inside. Despite this, it is far fewer $D>100 \mathrm{~km}$ craters than either one (Strom 1987), probably because Titania was extensively resurfaced at an unknown time (Croft \& Soderblom 1991). Our results indicate that time would be $t \sim 850 \mathrm{Myr}$ or roughly $3 \mathrm{Ga}$ (Figure 12). The source of geological activity on Titania at such relatively recent times is unknown.

We conclude by pointing out that the flux of projectiles and dark dust from the irregular satellites give us a new and potentially powerful tool for determining the chronology of events on the regular satellites. They also allow us to link these events to those happening elsewhere via the Nice model framework. By knitting all such events together, we hope to eventually create a tapestry that can accurately describe the history of the solar system.

We thank Scott Sheppard for his comments and for the use of his irregular satellite database. Additional helpful comments on this work were provided by Bonnie Buratti, Clark Chapman, Luke Dones, Michelle Kirchoff, Hal Levison, Jeff Moore, and Paul Schenk. We also thank Matjia Ćuk for his careful and constructive review. Research funds for William Bottke were provided by NASA's Lunar Science Institute and the Origins of Solar Systems Programs. Those for David Nesvorný were provided for by NSF's Planetary Astronomy program and NASA's Outer Planets Research Program. The work of David Vokrouhlický was partially supported by research grant 205/08/0064 of the Czech Grant Agency and the Research Program MSM0021620860 of the Czech Ministry of Education.

\section{REFERENCES}

Agnor, C. B., \& Hamilton, D. P. 2006, Nature, 441, 192

Astakhov, S. A., Burbanks, A. D., Wiggins, S., \& Farrelly, D. 2003, Nature, 423, 264

Banfield, D., \& Murray, N. 1992, Icarus, 99, 390

Barnes, J. W., et al. 2008, Icarus, 195, 400

Barr, A. C., \& Canup, R. M. 2010, Nature Geosciences, in press

Beaugé, C., \& Nesvorný, D. 2007, AJ, 133, 2537

Beaugé, C., Nesvorný, D., \& Dones, L. 2006, AJ, 131, 2299

Bell, J. F., Cruikshank, D. P., \& Gaffey, M. J. 1985, Icarus, 61, 192

Benz, W., \& Asphaug, E. 1999, Icarus, 142, 5

Bernstein, G. M., Trilling, D. E., Allen, R. L., Brown, M. E., Holman, M., \& Malhotra, R. 2004, AJ, 128, 1364

Bottke, W. F., Durda, D. D., Nesvorný, D., Jedicke, R., Morbidelli, A., Vokrouhlický, D., \& Levison, H. F. 2005a, Icarus, 179, 63

Bottke, W. F., Durda, D. D., Nesvorný, D., Jedicke, R., Morbidelli, A., Vokrouhlický, D., \& Levison, H. 2005b, Icarus, 175, 111

Bottke, W. F., Levison, H. F., Nesvorný, D., \& Dones, L. 2007, Icarus, 190, 203

Bottke, W. F., Morbidelli, A., Jedicke, R., Petit, J.-M., Levison, H. F., Michel, P., \& Metcalfe, T. S. 2002, Icarus, 156, 399

Bottke, W. F., Nolan, M. C., Greenberg, R., \& Kolvoord, R. A. 1994, Icarus, 107,255

Bottke, W. F., Vokrouhlický, D., Brož, M., Nesvorny, D., \& Morbidelli, A. 2001, Science, 294, 1693

Brasser, R., Morbidelli, A., Gomes, R., Tsiganis, K., \& Levison, H. F. 2009, A\&A, 507, 1053

Brown, R. H., et al. 2006, A\&A, 446, 707

Brož, M., \& Vokrouhlický, D. 2008, MNRAS, 390, 715

Buratti, B. J., Hicks, M. D., Tryka, K. A., Sittig, M. S., \& Newburn, R. L. 2002, Icarus, 155,375

Buratti, B. J., \& Mosher, J. A. 1991, Icarus, 90, 1

Buratti, B. J., \& Mosher, J. A. 1995, Icarus, 115, 219

Burns, J. A. 1986, in Satellites, ed. J. A. Burns \& M. S. Matthews (Tucson, AZ: Univ. Arizona Press), 117

Burns, J. A., Hamilton, D. P., Mignard, F., \& Soter, S. 1996, in ASP Conf. Ser. 104, IAU Colloq. 150, Physics, Chemistry, and Dynamics of Interplanetary Dust, ed. B. A. S. Gustafson \& M. S. Hanner (San Francisco, CA: ASP), 179 Burns, J. A., Lamy, P. L., \& Soter, S. 1979, Icarus, 40, 1 
Carruba, V., Burns, J. A., Nicholson, P. D., \& Gladman, B. J. 2002, Icarus, 158, 434

Carruba, V., Burns, J. A., Nicholson, P. D., \& Gladman, B. J. 2003, Icarus, 162, 230

Chapman, C. R., \& McKinnon, W. B. 1986, in Satellites, ed. J. A. Burns \& M. S. Matthews (Tucson, AZ: Univ. Arizona Press), 492

Charnoz, S., Morbidelli, A., Dones, L., \& Salmon, J. 2009, Icarus, 199, 413

Christou, A. A. 2005, Icarus, 174, 215

Colombo, G., \& Franklin, F. A. 1971, Icarus, 15, 186

Croft, S. K., \& Soderblom, L. A. 1991, in Uranus, ed. J. T. Bergstralh, E. D. Miner, \& M. S. Matthews (Tucson, AZ: Univ. Arizona Press) 561

Cruikshank, D. P., Bell, J. F., Gaffey, M. J., Brown, R. H., Howell, R., Beerman, C., \& Rognstad, M. 1983, Icarus, 53, 90

Cuk, M., \& Burns, J. A. 2004, Icarus, 167, 369

Ćuk, M., \& Gladman, B. J. 2006, Icarus, 183, 362

Davis, D. R., Durda, D. D., Marzari, F., Campo Bagatin, A., \& Gil-Hutton, R. 2002, in Asteroids III, ed. W. F. Cellino, A. Bottke, Jr., P. Paolicchi, \& R. P. Binzel (Tucson, AZ: Univ. Arizona Press), 545

Denk, T., \& Spencer, J. R. 2008, BAAS, 40, 510

Dohnanyi, J. S. 1969, J. Geophys. Res., 74, 2431

Dones, L., Weissman, P. R., Levison, H. F., \& Duncan, M. J. 2004, in Comets II, ed. M. C. Festou, H. U. Keller, \& H. A. Weaver (Tucson, AZ: Univ. Arizona Press), 153

Duncan, M. J., Levison, H. F., \& Lee, M. H. 1998, AJ, 116, 2067

Durda, D. D., Bottke, W. F., Enke, B. L., Merline, W. J., Asphaug, E., Richardson, D. C., \& Leinhardt, Z. M. 2004, Icarus, 170, 243

Durda, D. D., Bottke, W. F., Nesvorný, D., Enke, B. L., Merline, W. J., Asphaug, E., \& Richardson, D. C. 2007, Icarus, 186, 498

Emelyanov, N. V. 2005, A\&A, 438, L33

Estrada, P. R., \& Mosqueira, I. 2006, Icarus, 181, 486

Fernández, J. A., \& Morbidelli, A. 2006, Icarus, 185, 211

Fernández, Y. R., Sheppard, S. S., \& Jewitt, D. C. 2003, AJ, 126, 1563

Gladman, B., et al. 2001, Nature, 412, 163

Goldreich, P., Murray, N., Longaretti, P. Y., \& Banfield, D. 1989, Science, 245 500

Gomes, R., Levison, H. F., Tsiganis, K., \& Morbidelli, A. 2005, Nature, 435, 466

Grav, T., \& Bauer, J. 2007, Icarus, 191, 267

Grav, T., \& Holman, M. J. 2004, ApJ, 605, L141

Grav, T., Holman, M. J., \& Fraser, W. C. 2004, ApJ, 613, L77

Grav, T., Holman, M. J., Gladman, B. J., \& Aksnes, K. 2003, Icarus, 166, 33

Haghighipour, N., \& Jewitt, D. 2008, AJ, 136, 909

Hamilton, D. P. 1997, BAAS, 29, 1010

Heppenheimer, T. A., \& Porco, C. 1977, Icarus, 30, 385

Holsapple, K. A., \& Housen, K. R. 2009, Lunar and Planetary Institute Science Conf., Abstracts, 40, 1936

Jacobson, R. A., et al. 2006, AJ, 132, 2520

Jaumann, R., \& Neukum, G. 2009, Lunar and Planetary Institute Science Conf. Abstracts, 40, 1641

Johnson, T. V., \& Lunine, J. I. 2005, Nature, 435, 69

Johnson, T. V., Soderblom, L. A., Mosher, J. A., Danielson, G. E., Cook, A. F., \& Kupperman, P. 1983, J. Geophys. Res., 88, 5789

Jewitt, D. 1991, in Comets in the Post-Halley Era, ed. R. Newburn, M. Neugebauer, \& J. Rahe (Dordrecht: Kluwer), 19

Jewitt, D., \& Haghighipour, N. 2007, ARA\&A, 45, 261

Jewitt, D., \& Sheppard, S. 2005, Space Sci. Rev., 116, 441

Kenyon, S. J., \& Bromley, B. C. 2001, AJ, 121, 538

Kirchoff, M. R., \& Schenk, P. M. 2009, Lunar and Planetary Institute Science Conf. Abstracts, 40, 2067

Kortenkamp, S. J. 2005, Icarus, 175, 409

Lacerda, P., \& Jewitt, D. C. 2007, AJ, 133, 1393

Lamy, P. L., Toth, I., Fernandez, Y. R., \& Weaver, H. A. 2004, in Comets II, ed. M. C. Festou, H. U. Keller, \& H. A. Weaver (Tucson, AZ: Univ. Arizona Press), 223

Leinhardt, Z. M., \& Stewart, S. T. 2009, Icarus, 199, 542

Levison, H. F., Bottke, W. F., Gounelle, M., Morbidelli, A., Nesvorny, D., \& Tsiganis, K. 2009, Nature, 460, 364

Levison, H. F., Dones, L., Chapman, C. R., Stern, S. A., Duncan, M. J., \& Zahnlc, K. 2001, Icarus, 151, 286

Levison, H. F., Duncan, M. J., Zahnle, K., Holman, M., \& Dones, L. 2000, Icarus, 143,415

Levison, H. F., Morbidelli, A., Dones, L., Jedicke, R., Wiegert, P. A., \& Bottke, W. F. 2002, Science, 296, 2212

Lissauer, J. J. 1985, J. Geophys. Res., 90, 11289

Lorenz, R. D., et al. 2006, Science, 312, 724

Lorenz, R. D., et al. 2008, Geophys. Res. Lett., 35, 2206
McKinnon, W. B., Chapman, C. R., \& Housen, K. R. 1991, in Uranus, ed. J. T. Bergstralh, E. D. Miner, \& M. S. Matthews (Tucson, AZ: Univ. Arizona Press), 629

McKinnon, W. B., \& Parmentier, E. M. 1986, in Satellites, ed. J. A. Burns \& M. S. Matthews (Tucson, AZ: Univ. Arizona Press), 718

Minton, D. A., \& Malhotra, R. 2009, Nature, 457, 1109

Moore, J. M., \& Pappalardo, R. T. 2008, AGU Fall Meeting Abstracts, 6

Moore, J. M., et al. 2004, in Jupiter. The Planet, Satellites and Magnetosphere, ed. F. Bagenal, T. E. Dowling, \& W. B. McKinnon (Cambridge: Cambridge Univ. Press), 397

Morbidelli, A., Bottke, W. F., Nesvorny, D., \& Levison, H. F. 2009a, Icarus, 204,558

Morbidelli, A., \& Crida, A. 2007, Icarus, 191, 158

Morbidelli, A., Levison, H. F., Bottke, W. F., Dones, L., \& Nesvorny, D. 2009b, Icarus, 202, 310

Morbidelli, A., Levison, H. F., Tsiganis, K., \& Gomes, R. 2005, Nature, 435, 462

Morbidelli, A., Tsiganis, K., Crida, A., Levison, H. F., \& Gomes, R. 2007, AJ, 134, 1790

Mothé-Diniz, T., \& Nesvorný, D. 2008, in Asteroids, Comets, Meteors, LPI Contributions, 1405,8031

Nesvorný, D., Alvarellos, J. L. A., Dones, L., \& Levison, H. F. 2003, AJ, 126, 398

Nesvorný, D., Beaugé, C., \& Dones, L. 2004, AJ, 127, 1768

Nesvorný, D., \& Vokrouhlický, D. 2009, AJ, 137, 5003

Nesvorný, D., Vokrouhlický, D., \& Morbidelli, A. 2007, AJ, 133, 1962

Nicholson, P. D., Ćuk, M., Sheppard, S. S., Nesvorny, D., \& Johnson, T. V. 2008 in The Solar System Beyond Neptune, ed. M. A. Barucci, H. Boehnhardt, D. P. Cruikshank, \& A. Morbidelli (Tucson, AZ: Univ. Arizona Press), 411

Noll, K. S., Levison, H. F., Grundy, W. M., \& Stephens, D. C. 2006, Icarus, 184, 611

Pappalardo, R. T., et al. 2004, in Jupiter. The Planet, Satellites and Magnetosphere, ed. F. Bagenal, T. E. Dowling, \& W. B. McKinnon (Cambridge: Cambridge Univ. Press), 363

Pollack, J. B., Burns, J. A., \& Tauber, M. E. 1979, Icarus, 37, 587

Pollack, J. B., Witteborn, F. C., Erickson, E. F., Strecker, D. W., Baldwin, B. J., \& Bunch, T. E. 1978, Icarus, 36, 271

Press, W. H., Teukolsky, S. A., Vetterling, W. T., \& Flannery, B. P. 1992, Numerical Recipes in C (Cambridge: Cambridge Univ. Press)

Richardson, D. C., Bottke, W. F., \& Love, S. G. 1998, Icarus, 134, 47

Schmedemann, N., Neukum, G., Denk, T., \& Wagner, R. 2009, Lunar and Planetary Institute Science Conf. Abstracts, 40, 1941

Sheppard, S. S., Jewitt, D., \& Kleyna, J. 2005, AJ, 129, 518

Sheppard, S. S., Jewitt, D., \& Kleyna, J. 2006, AJ, 132, 171

Sheppard, S. S., \& Trujillo, C. A. 2008, BAAS, 40, 465

Soter, S. 1974, in IAU Colloq. 28, Brightness of Iapetus (New York: Cornel Univ.)

Spencer, J. R., Pearl, J. C., Segura, M., \& Cassini CIRS Team 2005, BAAS, 37, 706

Stöffler, D., \& Ryder, G. 2001, Space Sci. Rev., 96, 9

Storrs, A. D., Dunne, C., Conan, J.-M., Mugnier, L., Weiss, B. P., \& Zellner, B. 2005, Icarus, 173,409

Strom, R. G. 1987, Icarus, 70, 517

Strom, R. G., Malhotra, R., Ito, T., Yoshida, F., \& Kring, D. A. 2005, Science, 309, 1847

Strom, R. G., Woronaw, A., \& Gurnis, M. 1981, J. Geophys. Res., 86, 8659

Stuart, J. S., \& Binzel, R. P. 2004, Icarus, 170, 295

Tsiganis, K., Gomes, R., Morbidelli, A., \& Levison, H. F. 2005, Nature, 435 459

Verbiscer, A., French, R., Showalter, M., \& Helfenstein, P. 2007, Science, 315 815

Veverka, J., Brown, R. H., \& Bell, J. F. 1991, in Uranus, ed. J. T. Bergstralh, E D. Miner, \& M. S. Matthews (Tucson, AZ: Univ. Arizona Press), 528

Vokrouhlický, D., Nesvorný, D., \& Levison, H. F. 2008, AJ, 136, 1463

Weidenschilling, S. J., Spaute, D., Davis, D. R., Marzari, F., \& Ohtsuki, K. 1997, Icarus, 128, 429

Weissman, P. R., Asphaug, E., \& Lowry, S. C. 2004, in Comets II, ed. M. C Festou, H. U. Keller, \& H. A. Weaver (Tucson, AZ: Univ. Arizona Press), 337

Whitman, K., Morbidelli, A., \& Jedicke, R. 2006, Icarus, 183, 101

Wieczorek, M. A., \& LeFeuvre, M. 2009, Icarus, 200, 358

Zahnle, K., Alvarellos, J. L., Dobrovolskis, A., \& Hamill, P. 2008, Icarus, 194, 660

Zahnle, K., Dones, L., \& Levison, H. F. 1998, Icarus, 136, 202

Zahnle, K., Schenk, P., Levison, H., \& Dones, L. 2003, Icarus, 163, 263

Zahnle, K., Schenk, P., Sobieszczyk, S., Dones, L., \& Levison, H. F. 2001, Icarus, 153,111 